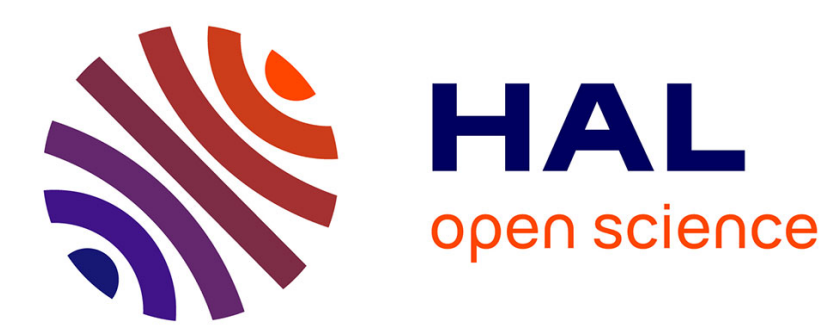

\title{
Orientation des productions bovines charolaises en Creuse
}

\author{
M. Lherm, D. Bebin, G. Lienard
}

\section{To cite this version:}

M. Lherm, D. Bebin, G. Lienard. Orientation des productions bovines charolaises en Creuse. Productions Animales, 1988, 1 (2), pp.97-109. hal-00895821

\section{HAL Id: hal-00895821 \\ https://hal.science/hal-00895821}

Submitted on 1 Jan 1988

HAL is a multi-disciplinary open access archive for the deposit and dissemination of scientific research documents, whether they are published or not. The documents may come from teaching and research institutions in France or abroad, or from public or private research centers.
L'archive ouverte pluridisciplinaire HAL, est destinée au dépôt et à la diffusion de documents scientifiques de niveau recherche, publiés ou non, émanant des établissements d'enseignement et de recherche français ou étrangers, des laboratoires publics ou privés. 


\section{LHERM, D. BEBIN G. LIENARD}

INRA Theix

Laboratoire d'Economie

de l'Elevage

63122 Ceyrat

\section{Orientation des productions bovine charolaises en Creuse.}

Dans les exploitations charolaises herbagères, faut-il vendre ses veaux en broutard, ou peut-on engraisser des taurillons à l'auge ou des jeunes taureaux de boucherie de 20 à 24 mois après une période de pâturage, et pour quels résultats économiques?

\begin{abstract}
Le choix d'un système de production en élevage allaitant charolais est difficile dans les zones herbagères. $\mathrm{S}$ les possibilités de production à partir du troupeau allaitant sont nombreuses, les éléments que l'éleveur doit prendre en compte le sont également. Il doit à la fois s'adapter aux conditions pédoclimatiques de son exploitation qui obligent, dans les zones charolaises du Centre de la France, à conserver une production d'herbe dominante, et tenir compte des conditions du marché des bovins-viande dont la demande est en évolution constante.
\end{abstract}

En vue d'apporter des éléments de réflexion, une trentaine d'élevages charolais du Nord-Est de la Creuse, pratiquant divers systèmes de production, ont été suivis aux plans technique et économique depuis 1981. Ces exploitations sont localisées autour d'un axe Boussac - Cheneraille - Aubusson. Elles sont représentatives de la diversité des conditions de milieu pédoclimatique que

\section{Résumé}

Les éleveurs de vaches allaitantes ont de nombreuses possibilités de production à partir de la vente en maigre ou de l'engraissement de leurs animaux.

Les choix doivent tenir compte de l'évolution des marchés et des systèmes fourragers possibles dans l'exploitation.

En région herbagère, comme la zone charolaise centrale, tous les systèmes de production ne sont pas également adaptés, comme le montre l'analyse comparée de trois échantillons d'éleveurs adhérents à un Groupement de Producteurs du Nord Est de la Creuse, et qui vendent leurs veaux mâles soit en broutards, soit après engraissement en taurillons à l'auge ou en jeunes taureaux de 20 ou 24 mois après une période de pâturage. Les meilleurs revenus sont, jusqu'à présent, obtenus avec cette dernière option qui est bien adaptée aux conditions des exploitations, car elle permet une meilleure utilisation des surfaces en herbe et des potentialités de la race charolaise. Elle est néanmoins exigeante en technicité et elle doit être parfaitement menée aux plans techniques et économiques.

Mais l'évolution de la conjoncture qui se dessine permettra-t-elle à l'avenir le maintien dans les zones allaitantes de cette production de jeunes bovins concurrencée par celle d'autres régions? rencontrent les éleveurs de la zone charolaise centrale, avec une prépondérance de sols humides ou en pente se prêtant mal aux labours, et avec des sécheresses estivales fréquentes. Une partie des exploitations est située en zone de Montagne, mais la majorité est en Piémont et surtout en Zone Défavorisée Simple.

Elles sont toutes adhérentes à un Groupement de Producteurs, essentiellement la coopérative des Producteurs de Bovins de la Creuse (CPBC). Une collaboration étroite a été établie avec ce Groupement qui se préoccupe beaucoup des conséquences des orientations qu'il peut contribuer à faire prendre à ses adhérents. L'étude a été menée en liaison avec les Services de la Chambre Départementale d'Agriculture et avec l'aide du FIDAR I.E. Massif Central.

Nous présentons ici, essentiellement, les résultats de la campagne 1986, sachant que les principales conclusions auxquelles peut aboutir la comparaison des différents systèmes analysés, sont constatées depuis le début de l'étude, malgré les caractéristiques particulières de l'année. L'évolution des résultats entre 1981 et 1985 a fait l'objet d'une publication antérieure (Lherm et al 1987). Rappelons que cette campagne a été marquée par la poursuite de la crise sur le marché de la viande bovine, qui sévit depuis 1983-84 à la suite de l'afflux de vaches laitières de réforme consécutif à la mise en place des quotas de production. En outre, une seconde sécheresse estivale a encore sévi, certes moins sévère et moins prolongée que celle de 1985 , mais dont les conséquences répétitives ont été graves pour les élevages de la zone charolaise.

\section{1 / Présentation de l'échantillon d'exploitations}

Parmi les 32 élevages suivis en 1986 :

- Neuf vendent la majorité des veaux mâles dès le sevrage en broutard $(\mathrm{Bt})$. 
- Onze engraissent la totalité ou la majorité des mâles à l'auge en taurillons vendus entre 15 et 18 mois d'âge (T18).

Les veaux sont mis à l'engraissement dès le sevrage, sur un régime à base d'ensilage de maïs complémenté en concentré. Les ventes s'effectuent après une période de finition de 6 à 10 mois, entre mai et septembre, selon les élevages.

- Six engraissent tout ou partie de leurs mâles en jeunes taureaux de 24 mois (T2a).

Les techniques de production sont bien au point (Micol 1986). Rappelons brièvement la façon dont elles sont mises en œuvre dans l'échantillon. Pendant le premier hiver qui suit le sevrage, les veaux ont une croissance de 600 à 800 g/jour, avec des régimes à base d'ensilage de maiis en quantité limitée et du foin ou de l'ensilage d'herbe (et des concentrés en faible quantité). Ils sont ressortis au pâturage non castrés et ne sont rentrés à l'étable qu'à l'automne, à l'âge de 18 mois, après avoir réalisé une croissance moyenne de 700 à 900 g/jour. Lengraissement final à l'auge dure de 3 à 5 mois selon les lots, avec de l'ensilage de maïs et du concentré. Les ventes ont lieu de janvier à début avril.

- Quatre engraissent tout ou partie de leurs mâles en jeunes taureaux de 20 mois (T20).

Ce système, plus rare, est proche du précédent mais la seconde saison de pâturage est écourtée. Les animaux sont rentrés en septembre, mais ils reçoivent parfois du concentré au pâturage. Ils sont engraissés plus rapidement et sont vendus entre octobre et fin décembre. Ce système est exigeant en technicité et il est préférable de disposer d'un silo de maïs "de report" pour démarrer l'engraissement (ce que tous n'ont pas encore réussi à réaliser).

Les deux dernières exploitations sont particulières. L'une a un système très diversifié (bovins, ovins, porcs) ; l'autre a vendu en 1986 ses mâles en maigre de 18 mois (non castrés), ayant renoncé à les engraisser comme habituellement (en T20).

- Par la suite nous utilisons les sigles (Bt, T18, T20, T2a) aussi bien pour les animaux produits que pour les exploitations correspondantes.

Le choix du système est quelque peu influencé par la localisation et les possibilités de production fourragère. En effet, les engraisseurs de T18 se retrouvent pour la plupart dans les zones les plus basses, aptes à produire du maiis fourrage et des céréales (région de Boussac) : 9 sont en zone défavorisée simple (ZDS) et 2 en Piémont. A l'opposé, les élevages de broutard sont en grande partie en altitude, 4 en Montagne, 3 en Piémont et 2 seulement en ZDS. Les producteurs de T2a sont en majorité en Piémont ( 1 seul en ZDS) et ceux de T20 plutôt en ZDS ( 3 et 1 en Piémont). Il s'agit en majorité d'exploitants assez jeunes ou ayant des successeurs (âge moyen 46 ans). Leur niveau technique moyen est bon et la plupart d'entre eux ont développé leur exploitation : 17 ont bénéficié d'un Plan de Développement ( 9 avant 1981 et 8 plus récemment). Les résultats observés dans ce groupe sont donc d'un niveau supérieur à la moyenne régionale. Ils ont l'intérêt de montrer les possibilités technico-économiques des systèmes.

Nous nous attacherons à faire ressortir les principaux faits, l'ensemble des résultats étant regroupés en annexe dans des tableaux détaillés se prêtant à une analyse complémentaire.

\section{2 / Les moyens de production et l'utilisation des surfaces}

(Tableau 1)

Avec 89 hectares de SAU et près de 2 UTH en moyenne, ces exploitations ont des moyens de production dépassant la moyenne régionale (1). $40 \%$ des terres sont en fermage, mais cette proportion varie selon les exploitations et notamment leur localisation, celles de plaine ayant moins de faire-valoir direct que celles de Piémont et Montagne. Les producteurs de broutard disposent des surfaces les plus restreintes (40 ha par UTH). Les engraisseurs ont, dans l'ensemble, plus de main-d'œuvre et de surface (avec près de 50 ha par UTH), ce qui rejoint une tendance générale. Les élevages produisant des $\mathrm{T} 20$ font un peu exception : ils ont moins de surface que les T2a, ce qui peut inciter au raccourcissement du cycle, mais ils ont aussi moins de main-d'œuvre, ce qui leur donne la plus grande surface par travailleur (54 ha/UTH).

La surface fourragère (SFP) est dominante, avec près de $85 \%$ de la SAU en moyenne ; mais les différences entre les systèmes sont nettes. Les exploitations qui vendent des broutards, localisées principalement en Montagne et Piémont, n'ont que $10 \%$ de surface en céréales. A l'opposé, celles produisant des T18, situées en majorité en plaine, ont $21 \%$ de céréales et autres cultures de vente (maïs et colza essentiellement). Celles qui engraissent des T20 et T2a ont des proportions intermédiaires.

Les systèmes fourragers sont différents, en harmonie avec les productions zootechniques. En production de broutard, la prairie naturelle est dominante ; 6 exploitations sur 9 font un peu de maïs ensilage, qui est réservé à l'engraissement des femelles parfois largement développé ; l'ensilage d'herbe est fréquent mais toujours

(1) La moyenne départementale est de 39 hectares en 1986, mais la zone charolaise de la Creuse a des surfaces supérieures à celles de la zone limousine.

Tableau 1. Les moyens de productions.

\begin{tabular}{|c|c|c|c|c|c|}
\hline $\begin{array}{l}\text { Système de production } \\
\text { Nombre d'exploitations }\end{array}$ & $\begin{array}{l}\text { Broutards } \\
\qquad 9\end{array}$ & $\begin{array}{l}\text { Taurillons } \\
18 \text { mois } \\
\text { gras } \\
11\end{array}$ & $\begin{array}{c}\text { Taureaux } \\
2 \text { ans } \\
\text { gras } \\
6\end{array}$ & $\begin{array}{c}\text { Taureaux } \\
20 \text { mois } \\
\text { gras } \\
4\end{array}$ & $\begin{array}{c}\text { Moyenne } \\
\text { du groupe } \\
\text { Creuse } \\
32\end{array}$ \\
\hline S.A.U (ha) & 72 & 94 & 101 & 80 & 89 \\
\hline U.T.H. & 1,83 & 1,97 & 2,09 & 1,46 & 1,97 \\
\hline UGB (Bovins+Ovins+Chevaux) & 81,9 & 84,4 & 109,0 & 85,6 & 91,0 \\
\hline UGB / UTH & 44,3 & 43,1 & 53,1 & 59,3 & 47,1 \\
\hline
\end{tabular}


Tableau 2. Résultats techniques du troupeau bovin.

\begin{tabular}{|l|c|c|c|c|c|}
\hline Système de production & Broutards & $\begin{array}{c}\text { Taurillons } \\
\mathbf{1 8} \text { mois } \\
\text { gras }\end{array}$ & $\begin{array}{c}\text { Taureaux } \\
\mathbf{2} \text { ans } \\
\text { gras }\end{array}$ & $\begin{array}{c}\text { Taureaux } \\
\mathbf{2 0} \text { mois } \\
\text { gras }\end{array}$ & $\begin{array}{c}\text { Moyenne } \\
\text { du groupe } \\
\text { creuse }\end{array}$ \\
\hline $\begin{array}{l}\text { Productivité en veau : } \\
\text { (ensemble du troupeau) }\end{array}$ & 93,1 & 92,3 & 92,3 & 92,6 & 92,9 \\
$\begin{array}{l}\text { - Productivité numérique (\%) } \\
\text { - Productivité globale (\%) } \\
\text { (nés + achetés) }\end{array}$ & 89,4 & 89,4 & 87,9 & 90,2 & 89,3 \\
$\begin{array}{l}\text { Date moyenne de vêlage } \\
\text { (charolais seulement) }\end{array}$ & $10 / 03$ & $28 / 02$ & $18 / 03$ & $18 / 02$ & $06 / 03$ \\
\hline
\end{tabular}

modéré ( 6 sur 9 et récoltent ainsi $10 \%$ de la SFP). Le maiis fourrage est beaucoup plus développé chez les T18 ; présent dans toutes les exploitations, il occupe $13 \%$ de la SFP. L'ensilage d'herbe est également présent dans 5 élevages sur 11 pour $15 \%$ de la SFP. Le système fourrager des T20 se rapproche de celui des T18 par l'importance donnée au maïs fourrage présent dans les 4 élevages sur près de $15 \%$ de la SFP et 2 sur 4 pratiquent l'ensilage d'herbe. En revanche, les producteurs de T2a ont un système fourrager différent : le maïs est toujours présent, en proportion inférieure de près de moitié à celle des deux autres types d'engraissement ; l'ensilage d'herbe n'est pas très développé et le foin reste dominant, mais l'accent a été mis sur les prairies temporaires qui occupent plus de la moitié de la SFP, et les techniques d'exploitation du pâturage sont bonnes.

Au total, on récolte plus de fourrages dans les systèmes T18 et T20 (47\% de la SFP soit 42 et 40 ares par UGB) que dans les systèmes broutard et T2a (43\% de la SFP soit 34 à 35 ares par UGB).

Le capital immobilisé varie peu entre les systèmes. Il est de l'ordre de $13000 \mathrm{~F}$ par ha pour le capital fixe (cheptel, matériel et bâtiments) ; avec les stocks et la trésorerie, l'ensemble atteint environ $14500 \mathrm{~F}$ par hectare et $14000 \mathrm{~F}$ par UGB. Il apparaît légèrement plus élevé (par hectare) pour les T2a, du fait qu'il y a davantage de cheptel par ha SAU (voir plus loin) et que la proportion de cheptel présent en fin d'exercice (au 31/12) est un peu plus importante que dans les autres systèmes, les jeunes taureaux étant proches de leur vente à cette date.

\section{3 / Des différences dans les résultats techniques et économiques des troupeaux bovins}

Analysons les différentes composantes qui concourrent au produit et à la marge par UGB, principaux indicateurs du fonctionnement économique des troupeaux.

\section{1 / La conduite du troupeau de mères est bonne dans l'ensemble}

(Tableau 2)

La majorité des vêlages a lieu pendant l'hiver $(73 \%$ durant le $1^{\text {er }}$ trimestre) et la proportion de vêlages très tardifs (au-delà du $1^{\text {er }}$ juin) est maintenant très réduite (moins de $2 \%$ ). La date moyenne se situe au 6 mars 1986 , soit 4 jours plus tôt qu'en 1985, car la sécheresse de 1985 n'a pas eu d'effets négatifs, n'étant intervenue qu'après la période de reproduction. D'ailleurs, les intervalles entre vêlages, 374 jours pour l'ensemble, sont inférieurs à la moyenne habituelle (377 jours).

La productivité en veau est excellente dans l'ensemble puisque la productivité numérique approche $93 \%$, grâce à une faible mortalité des veaux $(6,4 \%)$, et la productivité globale dépasse $89 \%$, ce qui est exceptionnel en charolais.

Les différences de productivité en veau sont faibles entre les groupes. Les meilleurs résultats se trouvent, heureusement, en production de broutards, notamment grâce à une mortalité réduite $(5 \%)$ ce qui est essentiel dans ce système. Les résultats sont plutôt moins bons dans les T18 où la mortalité est la plus élevée $(8,4 \%)$, sans doute du fait de cheptels dans l'ensemble mieux conformés. En revanche, les T18 ont, avec les T20, les vêlages les plus précoces (car, situés en plaine, la mise à l'herbe peut s'y faire un peu plus tôt). Les mises-bas les plus tardives se trouvent dans les élevages de T2a, ce qui est plus admissible car c'est là où l'incidence est la plus faible, le retard pouvant être plus facilement rattrapé sur une durée d'élevage plus longue.

Globalement, on observe de bons résultats d'ensemble, ce qui laisse peu de place à une amélioration possible. Tout le problème est de les maintenir !

\section{2 / Caractéristiques des mâles à la vente} (Tableau 3)

\section{a / Production de broutards : trop légers, les ani- maux ont été pénalisés par la sécheresse.}

Si la majorité des veaux est toujours vendue à l'automne, les éleveurs se mettent progressivement à en conserver une partie pour les vendre en fin d'hiver, en "broutard de report". En 1986, 22 \% des broutards sont ainsi vendus après le $1^{\text {er }}$ janvier. Les broutards d'automne ont été vendus à $280 \mathrm{~kg}$ de poids vif en moyenne (à 7,3 mois d'âge). Ce poids est en forte baisse par rapport à 1985 (- $15 \mathrm{~kg}$ environ) du fait de la sécheresse de l'automne 1986. En revanche, les prix de vente $(16,90 \mathrm{~F} / \mathrm{kg})$ ont augmenté d'environ $0,40 \mathrm{~F} / \mathrm{kg}$, ce qui limite la perte par tête à $110-120 \mathrm{~F}(-2,5 \%$ environ, en francs courants). Les broutards "de report" vendus début 1986 sont plus lourds que ceux d'automne, sans être exceptionnels $(323 \mathrm{~kg})$ et ils sont également un peu plus légers $(-3 \mathrm{~kg})$ que ceux de l'année précédente, conséquence cette fois de la sécheresse de 1985.

\section{b / Production de taurillons de 18 mois : une amélioration.}

Les T18 représentent les $3 / 4$ du total des mâles commercialisés. Les autres sont vendus essentiellement en
Dans l'ensemble, la productivité en veau est excellente avec une productivité numérique proche de $93 \%$. 
Tableau 3. Caractéristiques des animaux vendus par système de production.

\begin{tabular}{|c|c|c|c|c|c|}
\hline \multicolumn{6}{|c|}{ ANIMAUX MALES } \\
\hline \multirow{2}{*}{$\begin{array}{l}\text { Système de production } \\
\text { et animaux vendus }\end{array}$} & \multicolumn{2}{|c|}{ Broutards } & \multirow{2}{*}{$\begin{array}{l}\text { Taurillons } \\
18 \text { mois } \\
\text { gras }\end{array}$} & \multirow{2}{*}{$\begin{array}{c}\text { Taureaux } \\
2 \text { ans } \\
\text { gras }\end{array}$} & \multirow{2}{*}{$\begin{array}{c}\text { Taureaux } \\
20 \text { mois } \\
\text { gras }\end{array}$} \\
\hline & $\begin{array}{l}\text { vente } \\
\text { automne }\end{array}$ & $\begin{array}{l}\text { vente } \\
\text { hiver }\end{array}$ & & & \\
\hline $\begin{array}{l}\text { Proportion (en nombre) } \\
\text { des ventes de mâles (\%) }\end{array}$ & 73 & 20 & 74 & 75 & 66 \\
\hline $\begin{array}{l}\text { Poids moyen }(\mathrm{kg}) \\
\text { (v : vif, } \mathrm{n}: \text { net) }\end{array}$ & $280 \mathrm{v}$ & $323 \mathrm{v}$ & $383 \mathrm{n}$ & \multirow{2}{*}{$\begin{array}{c}409 n \\
23,08 n\end{array}$} & \multirow{2}{*}{$\begin{array}{c}402 \mathrm{n} \\
23,08 \mathrm{n}\end{array}$} \\
\hline Prix en $\mathrm{F} / \mathrm{kg}$ & $16,91 \mathrm{v}$ & $16,29 \mathrm{v}$ & $22,80 \mathrm{n}$ & & \\
\hline \multicolumn{6}{|c|}{ VACHES DE RÉFORME ETT GÉNISSES } \\
\hline Système de production & \multicolumn{2}{|c|}{ Broutards } & $\begin{array}{l}\text { Taurillons } \\
18 \text { mois }\end{array}$ & $\begin{array}{l}\text { Taureaux } \\
2 \text { ans }\end{array}$ & $\begin{array}{l}\text { Taureaux } \\
20 \text { mois }\end{array}$ \\
\hline \multirow{3}{*}{$\begin{array}{l}\text { Vaches de réforme : } \\
\text { - Prix (en F/tête) } \\
\text { - Proportion (en nombre) } \\
\text { de vaches de réforme } \\
\text { engraissées (\%) } \\
\text { - Poids net des } \\
\text { vaches engraissées (kg) }\end{array}$} & \multicolumn{2}{|c|}{6968} & 8781 & 7677 & 9385 \\
\hline & \multicolumn{2}{|c|}{56} & 96 & 60 & 94 \\
\hline & \multicolumn{2}{|c|}{366} & 392 & 393 & 399 \\
\hline $\begin{array}{l}\text { Proportion (en nombre) } \\
\text { de génisses engraissées (\%) }\end{array}$ & \multicolumn{2}{|c|}{30} & 74 & 56 & 88 \\
\hline
\end{tabular}

broutard, afin d'homogénéiser les lots et de s'ajuster aux réserves fourragères.

Le poids moyen de carcasse (383 $\mathrm{kg}$ net à 16,5 mois) est supérieur à ceux des années précédentes qui étaient très faibles (368 kg en 1985). Ce changement provient à la fois d'une amélioration réelle $(+13 \mathrm{~kg}$ sur échantillon constant) et du fait qu'en 1986 nous avons suivi de nouveaux élevages choisis parmi les plus performants de la $\mathrm{CPBC}$. Bien que les prix aient légèrement baissé, de près de $0,30 \mathrm{~F} / \mathrm{kg}$ net $(-1 \%)$, on gagne globalement $150 \mathrm{~F}$ par tête.

Certaines exploitations vendent toujours des taurillons jeunes (14-15 mois) à des dates précoces (mai-juin). Les autres vendent normalement des animaux plus âgés, de juillet à septembre, avec un certain avancement en 1986 à la suite d'une demande pour des ventes en vif. Un exploitant qui fait naître la moitié des veaux en novembre-décembre vend en avril des taurillons de 17 mois et un autre vend en fin d'année des animaux âgés de 20 mois. Tout ceci concourt à un étalement réel des ventes, sans que cela influe beaucoup sur les marges unitaires (les ventes d'animaux jeunes s'accompagnent souvent de charges plus faibles, et le chargement n'est pas moindre, le système s'étant adapté).

\section{c / Production de jeunes taureaux de 2 ans et 20 mois : moins lourds du fait de la sécheresse et de la demande du marché.}

Chez les producteurs de T2a, $75 \%$ des mâles sont vendus dans cette catégorie ; $12 \%$ sont vendus en taurillon d'auge, du fait d'une exploitation qui vend la moitié des veaux (les plus lourds) en T18 et l'autre moitié en T2a. Le reste est vendu en broutard de fin d'hiver. Le poids moyen des $\mathrm{T} 2 \mathrm{a}$ (409 $\mathrm{kg}$ net à 23,7 mois) reste correct mais il est inférieur à celui de $1985(-24 \mathrm{~kg}$, avec il est vrai 20 jours d'âge en moins). Les ventes ont été réalisées plus tôt, au 10 mars 1986 au lieu du 28 mars 1985, du fait des plus faibles disponibilités en maiis ensilage, et surtout de la pression du marché recherchant des carcasses plus légères. Les prix de vente (23,08 F par kilo net) sont aussi en baisse d'environ $0,28 \mathrm{~F}$ par kilo $(-1,2 \%$ ), ce qui entraine une baisse de plus de $600 \mathrm{~F}$ par tête $(-6 \%)$.

Chez les producteurs de T20, il y a très peu de tri et la majorité des veaux est engraissée. Mais ils se commercialisent en 2 lots : les premiers, les plus nombreux, sont effectivement vendus à l'automne en T20, en moyenne au 29 octobre, à un poids de $402 \mathrm{~kg}$ et à 20,6 mois d'âge ; les seconds plus légers sont des T2a vendus en début d'hiver (12 février) à $395 \mathrm{~kg}$ net et 22,7 mois d'âge. Le prix des T20 $(23,08 \mathrm{~F} / \mathrm{kg})$ n'a guère été plus élevé que celui des T18 vendus en été $(22,79 \mathrm{~F} / \mathrm{kg}$ pour l'ensemble des 32 élevages), contrairement à ce qu'on pouvait espérer.

Au total, les poids des broutards et des T2a ont baissé du fait de la sécheresse. Ils se sont améliorés en T18. Les évolutions de prix ont favorisé les broutards et pénalisé l'engraissement.

\section{3 / Les femelles vendues}

\section{(Tableau 3)}

L'engraissement des femelles est très développé dans cet échantillon. Près de $55 \%$ des génisses non conservées pour le renouvellement et $75 \%$ des vaches de réforme sont engraissées. C'est le résultat de l'incitation du groupement de producteurs. Cet engraissement se retrouve dans tous les systèmes, avec naturellement des différences. Les producteurs de broutards engraissent moins de génisses ( 3 seulement sur 9 le font systématiquement) et la moitié seulement de leurs vaches. Alors que les engraisseurs finissent presque toutes leurs vaches et plus des $3 / 4$ de leurs génisses ( $92 \%$ chez les T20 !). 
Le poids moyen de carcasse de vaches $(387 \mathrm{~kg})$ est légèrement plus faible qu'en 1985 ( $-9 \mathrm{~kg})$, en partie du fait des sécheresses successives, mais les prix diminuent nettement $(-0,86 \mathrm{~F} / \mathrm{kg}$ net, soit $-4 \%)$, et pour l'ensemble des vaches, engraissées ou non, la réduction de prix est de $634 \mathrm{~F}$ par tête $(-7,5 \%$, ce qui est considérable et peu encourageant). Les meilleures ven- . tes de vaches se retrouvent chez les T18 $(8790 \mathrm{~F}$ de moyenne) et surtout les T20 (9 $385 \mathrm{~F}$... contre $6918 \mathrm{~F}$ en broutard). De même, si le poids des génisses engraissées de diverses catégories (24-30-36 mois se maintient ( $+3 \mathrm{~kg}$ net) notamment celui des 30 mois vendues en été ( $355 \mathrm{~kg}$ à 31.3 mois) malgré la sécheresse, leurs prix de vente diminuent également de $0,78 \mathrm{~F} / \mathrm{kg}$ net $(-3 \%)$ et de $200 \mathrm{~F}$ par tête.

Au total, les poids des femelles engraissées se maintiènnent, ou presque, malgré la sécheresse, mais les prix de vente chutent fortement, contribuant à réduire les marges laissées par les troupeaux.

\section{4 / L'équilibre général des ventes}

L'indicateur le plus synthétique de l'orientation des systèmes de production est la proportion des ventes d'animaux engraissés. En considérant l'ensemble des mâles et des femelles, celle-ci atteint, en valeur, $71 \%$ du total pour l'ensemble des élevages. Ce qui est exceptionnel dans ces zones herbagères où la production de maigre domine. Cette proportion atteint $96 \%$ chez les T20, $88 \%$ chez les T18 et $83 \%$ chez les T2a, contre $29 \%$ pour les producteurs de broutards.

\section{5 / Une productivité pondérale des troupeaux qui réserve quelques surprises}

(Tableau 4)

La productivité pondérale des troupeaux peut s'exprimer par le poids de viande (en $\mathrm{kg}$ vif) produit par UGB production "brute", dont il faut déduire les kg correspondant à la valeur des concentrés consommés (minéraux inclus) et des fourrages grossiers achetés, ce qui correspond à la production "autonome". Il faut noter que les achats de fourrages grossiers ont été, en 1986 comme en 1985, plus importants qu'en moyenne, tout comme la consommation de concentré (voir plus loin).
Pour l'ensemble des élevages, on observe :

- $298 \mathrm{~kg}$ de production de viande "brute" par UGB Bovin

- $237 \mathrm{~kg}$ de production de viande automone par UGB Bovin.

Sur échantillon constant, la productivité brute est maintenue en 1986 par rapport à 1985 (- $1 \mathrm{~kg}$ ) mais elle diminue de $13 \mathrm{~kg}$ par rapport à une année "normale" comme 1984. La productivité autonome baisse de $5 \mathrm{~kg}$, et de $23 \mathrm{~kg}$ par rapport à 1984, résultat de deux sécheresses consécutives mais aussi de l'évolution du marché qui recherche des animaux plus légers.

Les productivités pondérales "brutes" sont plus élevées en T18 $(325 \mathrm{~kg})$ et en T20 (329 kg), qu'en T2a $(293 \mathrm{~kg})$ et qu'en broutard $(254 \mathrm{~kg})$. Mais les écarts se creusent nettement entre les 3 systèmes d'engraissement lorsqu'on tient compte des concentrés distribués et des achats de fourrage : la productivité autonome reste la plus élevée pour les T18 réputés les plus intensifs $(258 \mathrm{~kg})$; les T2a sont très proches $(253 \mathrm{~kg})$ mais la productivité des T20 diminue à $246 \mathrm{~kg}$ du fait d'une exploitation qui emploie beaucoup trop de concentrés. La productivité autonome des élevages de broutards tombe à $201 \mathrm{~kg}$ par UGB, malgré l'excellente productivité en veau et l'engraissement de la moitié des vaches.

\section{6 / Des écarts de productivité économique plus importants encore}

La productivité économique des troupeaux est appréciée par la marge bovine finale par UGB.

Celle-ci atteint en moyenne pour les 32 élevages, $2591 \mathrm{~F}$ par UGB. Par rapport à 1985 sur l'échantillon constant, elle baisse de $242 \mathrm{~F}$ par UGB, soit $8,6 \%$ en francs courants et $11 \%$ en francs constants, ce qui est considérable. Cela provient à la fois de la diminution du produit $(-140 \mathrm{~F})$ et de la hausse des charges proportionnelles $(+61 \mathrm{~F}$ de charges du troupeau, entièrement dues au concentré et $+41 \mathrm{~F}$ de charges de production fourragère).

Les meilleurs résultats sont obtenus, comme les années antérieures par les producteurs de T2a, avec $2907 \mathrm{~F}$ par UGB, essentiellement grâce à la modération des charges engagées, tant celles relatives au troupeau

\section{Les producteurs de taurillons obtiennent des résultats décevants : le produit brut est élevé mais les charges le sont aussi, tant celles du troupeau que celles de la production fourragère.}

Tableau 4. Résultats économiques du troupeau bovin et de la surface fourragère.

\begin{tabular}{|c|c|c|c|c|c|}
\hline Système de production & Broutards & $\begin{array}{l}\text { Taurillons } \\
18 \text { mois } \\
\text { gras }\end{array}$ & $\begin{array}{c}\text { Taureaux } \\
2 \text { ans } \\
\text { gras }\end{array}$ & $\begin{array}{c}\text { Taureaux } \\
20 \text { mois } \\
\text { gras }\end{array}$ & $\begin{array}{l}\text { Moyenne } \\
\text { du groupe } \\
\text { Creuse }\end{array}$ \\
\hline Résultats du troupeau bovin : & & & & & \\
\hline Produit brut (en F/UGBB) & 3784 & 4484 & 4069 & 4725 & 4214 \\
\hline $\begin{array}{l}\text { Marge bovine finale } \\
\text { (en F/UGBB) }\end{array}$ & 2299 & 2642 & 2907 & 2720 & 2591 \\
\hline $\begin{array}{l}\text { Production de viande autonome } \\
\text { (kg/UGBB) }\end{array}$ & 201 & 258 & 253 & 246 & 237 \\
\hline $\begin{array}{l}\text { Résultats de la surface } \\
\text { fourragère : }\end{array}$ & & & & & \\
\hline Chargement (par ha SFP) & 1,24 & 1,16 & 1,24 & 1,20 & 1,21 \\
\hline Marge brute (en F/ha SFP) (1) & 2845 & 3128 & 3611 & 3299 & 3163 \\
\hline $\begin{array}{l}\text { Production de viande autonome } \\
\text { (kg/ha SFP) }\end{array}$ & 251 & 300 & 316 & 296 & 288 \\
\hline
\end{tabular}

(1) Frais de culture de la surface fourragère déduits. Ensemble des production de la SFP (bovins, ovins, chevaux, divers) 
(784 F dont $448 \mathrm{~F}$ de concentré, soit $344 \mathrm{~kg}$ par UGB), que celles relatives à la production fourragère.

Viennent ensuite les producteurs de $\mathrm{T} 20$, avec $2720 \mathrm{~F}$ par UGB. Ils obtiennent le meilleur produit mais les charges sont très élevées, notamment en concentré (680 kg et $926 \mathrm{~F}$ par UGB, particulièrement du fait de l'élevage qui complémente ses taurillons pendant toute la saison de pâturage et qui les finit au pré, ce qui est peu rationnel).

Les producteurs de T18 obtiennent des résultats un peu décevants avec $2642 \mathrm{~F}$ de marge par UGB, ce qui ne dépasse que de $340 \mathrm{~F}$ celle des producteurs de broutard (2 299 F) ! En effet, si le produit est nettement plus élevé, les charges le sont aussi, tant en ce qui concerne le troupeau avec $1247 \mathrm{~F}$ (dont $819 \mathrm{~F}$ et $631 \mathrm{~kg}$ de concentré) que pour la production fourragère (notamment du fait de l'importance du maïs). Les frais vétérinaires sont aussi relativement lourds tout comme chez les T20, ce qui est à relier à la mortalité des veaux et aux difficultés de vêlages plus fréquentes.

En conclusion, les résultats économiques obtenus par unité de cheptel sont les meilleurs pour les producteurs de taureaux de 2 ans, grâce à la modération des charges. Ils sont les plus faibles pour les éleveurs de broutards du fait de la modicité du produit. Mais ils sont très décevants pour les engraisseurs de taurillons à l'auge dont les charges de production sont très lourdes. Cette constatation est la même depuis le début de l'étude en 1981.

\section{4 / Productivité des surfaces fourragères et du travail : les écarts s'accentuent encore}

Malgré un système fourrager plus intensifié, comportant une forte proportion de mais fourrage $(13 \%)$ et les plus lourdes dépenses (700 F par ha SFP), les engraisseurs de T18 ont les chargements en cheptel les plus faibles (1,16 UGB par ha SFP). La production pondérale autonome ne dépasse pas $300 \mathrm{~kg}$ par ha SFP et la marge (ovins inclus) est égale à $3128 \mathrm{~F}$ en moyenne.

Avec un chargement de 1,24 UGB par ha SFP, et la meilleure marge par UGB, les producteurs de T2a obtiennent les marges par hectare SFP les plus élevées ( $3611 \mathrm{~F}$ avec $316 \mathrm{~kg}$ de viande vive autonome). Cela dépasse quelque peu les résultats des producteurs de T20 qui, avec un chargement plus faible $(1,20)$ et une marge par UGB inférieure, réalisent une marge de 3299 F par ha SFP (296 kg de viande autonome), résultat susceptible d'amélioration.

Malgré un chargement très correct $(1,24)$, les producteurs de broutards obtiennent les résultats par hectare SFP les plus faibles ( $2845 \mathrm{~F}$ et $251 \mathrm{~kg}$ de viande autonome), du fait des performances limitées par unité de cheptel.

Néanmoins, ce sont les résultats par travailleur qui sont les plus significatifs.

Les producteurs de $\mathrm{T} 2 \mathrm{a}$ et $\mathrm{T} 20$ arrivent à la meilleure productivité avec respectivement 53 et 59 UGB par UTH. Dans les deux cas, la marge de la surface fourragère dépasse $150000 \mathrm{~F}$ par travailleur. Ce sont des valeurs élevées en élevage allaitant.

En revanche, les producteurs de T18 et de broutard obtiennent des résultats nettement plus faibles, $115000 \mathrm{~F}$ par UTH pour les premiers et $100000 \mathrm{~F}$ pour les seconds, ceci pour des raisons différentes. Dans le cas du broutard, les plus faibles surfaces conduisent à un cheptel réduit (44 UGB par UTH) malgré le chargement correct et la forte spécialisation fourragère ( $90 \%$ de SFP dans la SAU), et la marge par UGB est, on l'a vu, la plus faible. Les résultats sont surprenants pour les engraiseurs de T18 : la conjonction d'un chargement relativement faible $(1,16)$ et d'une forte proportion de cultures céréalières limite l'importance du troupeau (43 UGB par UTH) et la marge par UGB est tout juste moyenne..

En conclusion, la marge du troupeau par unité de cheptel et le nombre d'UGB par travailleur discriminent nettement les systèmes. Les systèmes d'engraissement basés sur une utilisation de l'herbe (T2a, T20) arrivent à de bons résultats, et dépassent $150000 \mathrm{~F}$ de marge SFP par travailleur. Les producteurs de broutards et de T18 ont des résultats inférieurs de près de $30 \%$.

Mais il faut tenir compte des autres productions, notamment des céréales pour les élevages de T18, et des charges de structure.

\section{5 / Résultats économiques globaux : des écarts de revenus importants}

(Tableaux 5, 6 et 7 )

Remarque préalable : les résultats économiques présentés comprennent toutes les aides (sauf les subventions d'équipement qui sont des ressources exceptionnelles). L'indemnité Sécheresse 1985, est incluse en 1986, année où elle a été reçue.

\section{1 / Les engraisseurs de taurillons}

Effectivement, les engraisseurs de T18 ont une marge céréalière par travailleur plus que double de celle des autres systèmes ; à la plus forte proportion de surface cultivée s'ajoutent de meilleurs rendements (37 qx par ha pour l'ensemble des céréales) et une meilleure marge par hectare de culture $(2110 \mathrm{~F}$ contre 1700 à $1800 \mathrm{~F}$ pour les autres groupes).

Néanmoins, cela ne suffit pas à rétablir l'équilibre, d'autant plus que les charges de structures sont les plus lourdes (150 à $400 \mathrm{~F}$ de plus par hectare par rapport aux autres groupes), en raison de charges de mécanisation mais surtout de charges financières et foncières plus élevées. Ces dernières résultent de la plus forte proportion de fermage et des tarifs plus lourds que la moyenne, tant pour les fermages que les impôts fonciers et les Allocations Familiales et Vieillesse liées au revenu cadastral, conséquence d'une localisation réputée plus favorable.

Au total, le revenu agricole est très bas, moins de $700 \mathrm{~F}$ de revenu agricole par hectare et $32000 \mathrm{~F}$ de revenu du travail et des capitaux propres (1) par travailleur (malgré $62 \%$ d'aide). La conséquence est un

(1) Critère qui homogénéise les résultats économiques au plan du fermage et du travail : revenu du travail et des capitaux propres d'exploitation : revenu agricole + salaires + avantages - charges supplétives foncières (valeur locative - impôt foncier).

(2) Cash flow : revenu agricole + ressources exceptionnelles (y compris les aides exceptionnelles, subventions d'équipement et autres) + amortissements : correspond à l'ensemble des ressources "disponibles" (brut) pour les remboursements + l'autofinancement de l'augmentation du capital d'exploitation sous toutes ses formes + les prélèvements privés. 
Tableau 5. Résultats économiques globaux.

\begin{tabular}{|c|c|c|c|c|c|}
\hline Système de production & Broutards & $\begin{array}{c}\text { Taurillons } \\
18 \text { mois } \\
\text { gras }\end{array}$ & $\begin{array}{c}\text { Taureaux } \\
2 \text { ans } \\
\text { gras }\end{array}$ & $\begin{array}{c}\text { Taureaux } \\
20 \text { mois } \\
\text { gras }\end{array}$ & $\begin{array}{l}\text { Moyenne } \\
\text { du groupe } \\
\text { Creuse }\end{array}$ \\
\hline \multicolumn{6}{|l|}{$\begin{array}{l}\text { Résultats économiques } \\
\text { globaux : }\end{array}$} \\
\hline - en F/ha SAU & & & & & \\
\hline Produit brut d'exploitation & 4792 & 5050 & 5022 & 5746 & 5035 \\
\hline Marge brute & 3228 & 3231 & 3774 & 3558 & 3363 \\
\hline $\begin{array}{l}\text { Charges } \\
\text { de structures réelles (1) }\end{array}$ & 2385 & 2534 & 2129 & 2277 & 2399 \\
\hline Revenu agricole & 844 & 698 & 1645 & 1281 & 964 \\
\hline - en F/travailleur & & & & & \\
\hline $\begin{array}{l}\text { Revenu du travail } \\
\text { et des capitaux (2) }\end{array}$ & 28310 & 31987 & 72902 & 51810 & 39722 \\
\hline $\begin{array}{l}\text { Total des charges de structure } \\
\text { réelles (F/ha SAU) }\end{array}$ & 2288 & 2488 & 2035 & 2131 & 2320 \\
\hline
\end{tabular}

(1) Charges de structure réelles + charges proportionnelles non affectables

(2) Revenu du travail et capitaux propres d'exploitation = Revenu agricole + salaires et avantages - Charge supplétive foncière (valeur locative impôt foncier).

endettement qui s'accroît sans cesse. Les remboursements d'emprunt (à long et moyen terme + court terme et dettes d'exploitation + annuités foncières) absorbent la quasi-totalité du cash-flow (2) et le revenu disponible (I) ne dépasse pas $17100 \mathrm{~F}$ par exploitation, malgré une légère décapitalisation sur les stocks. Certains investissements en équipement ayant été faits (dont le total dépasse les amortissements), il est clair que ceux-ci n'ont pu être financés que par emprunts nouveaux et les exploitations ayant les plus faibles revenus se sont endettées pour vivre. De ce fait, l'endettement LMT d'exploitation augmente de $23000 \mathrm{~F}$ et le CT de $30000 \mathrm{~F}$, en moyenne.

\section{2 / Les éleveurs de broutards}

La marge globale obtenue par hectare (aides comprises) est du même ordre que celle des T18. Les charges de structure sont presque aussi élevées ; cela provient en partie de la surface plus réduite qui alourdit les frais de mécanisation (près de $800 \mathrm{~F}$ par ha, pour un système peu intensif). En revanche, les charges foncières sont plus faibles car la proportion de fermage est réduite et les tarifs sont les moins lourds du fait de la localisation en montagne et Piémont. En outre, les frais financiers sont modérés car certains élevages sont très peu endettés.
Dans ces conditions, les revenus sont les plus faibles, avec $844 \mathrm{~F}$ de revenu agricole par ha et $28300 \mathrm{~F}$ de revenu du travail et capitaux propres par UTH. Les aides (avec l'indemnité sécheresse 1985) dépassent le revenu.

L'endettement moins élevé ( $21 \%$ sur capital réduit, contre $41 \%$ pour les T18) limite les remboursements qui absorbent néanmoins la moitié d'un cash-flow modeste. Du fait d'une décapitalisation du cheptel (qui accroît les rentrées d'argent), le disponible I approche $71000 \mathrm{~F}$ par exploitation. Les nouveaux investissements en équipement sont équivalents aux amortissements, le nouvel endettement d'exploitation est légèrement inférieur aux remboursements, mais cela n'est possible que parce que les prélèvement familiaux et l'épargne (disponible II) sont très modestes en moyenne (53 $500 \mathrm{~F}$ par exploitation pour 1,7 UTH familial, $2600 \mathrm{~F}$ par mois...).

\section{3 / Les producteurs de jeunes taureaux de boucherie}

Chez les producteurs de T20, la marge globale est plus élevée par hectare que dans les groupes précédents. Malgré des frais financiers importants, les charges de structure sont plus faibles que celles des producteurs de T18,

Tableau 6. Endettement.

\begin{tabular}{|l|r|r|r|r|r|}
\hline Système de production & Broutards & $\begin{array}{c}\text { Taurillons } \\
\text { 18 mois } \\
\text { gras }\end{array}$ & $\begin{array}{c}\text { Taureaux } \\
\text { 2 ans } \\
\text { gras }\end{array}$ & $\begin{array}{c}\text { Taureaux } \\
\text { 20 mois } \\
\text { gras }\end{array}$ & $\begin{array}{c}\text { Moyenne } \\
\text { du groupe } \\
\text { Creuse }\end{array}$ \\
\hline $\begin{array}{l}\text { Capital d'exploitation (1) } \\
\text { au 31/12 en F/exploitation } \\
\begin{array}{l}\text { Taux d'endettement sur capital } \\
\text { réduit (hors foncier) } \\
\text { Remboursement dans l'exercice } \\
\text { (en F/exploitation) }\end{array}\end{array}$ & 1007049 & 1051810 & 1462388 & 1028374 & 1146312 \\
\hline
\end{tabular}

(1) Capital réduit $=$ Capital cheptel + Matériel + Batiment (non compris les stocks, valeurs en terre et liquidités).

Dans les exploitations où elle est pratiquée, la production de taureaux de boucherie de 2 ans conduit aux meilleurs résultats économiques. 
Tableau 7. Utilisation du revenu et financement des investissements (en francs par exploitation).

\begin{tabular}{|c|c|c|c|c|c|}
\hline Système de production & Broutards & $\begin{array}{l}\text { Taurillons } \\
18 \text { mois } \\
\text { gras }\end{array}$ & $\begin{array}{c}\text { Taureaux } \\
2 \text { ans } \\
\text { gras }\end{array}$ & $\begin{array}{l}\text { Taureaux } \\
20 \text { mois } \\
\text { gras }\end{array}$ & $\begin{array}{c}\text { Moyenne } \\
\text { du groupe } \\
\text { Creuse }\end{array}$ \\
\hline $\begin{array}{l}\text { Revenu agricole } \\
\text { dont aides (en \%) }\end{array}$ & $\begin{array}{r}56869 \\
105 \\
\end{array}$ & $\begin{array}{r}72750 \\
64 \\
\end{array}$ & $\begin{array}{r}164978 \\
37 \\
\end{array}$ & $\begin{array}{r}91176 \\
52 \\
\end{array}$ & $\begin{array}{r}85637 \\
63 \\
\end{array}$ \\
\hline Cash-Flow & 105269 & 124091 & 230554 & 133498 & 142786 \\
\hline $\begin{array}{l}\text { Variations des stocks de } \\
\text { production } \\
\text { Variation stocks aliments } \\
+ \text { engrais }\end{array}$ & $\begin{array}{r}-17197 \\
2588 \\
\end{array}$ & $\begin{array}{r}+279 \\
-9807 \\
\end{array}$ & $\begin{array}{r}+47500 \\
2956 \\
\end{array}$ & $\begin{array}{l}-27500 \\
-14225 \\
\end{array}$ & $\begin{array}{l}-1839 \\
-3251 \\
\end{array}$ \\
\hline $\begin{array}{l}\text { Remboursements } \\
\text { d'emprunts (1) } \\
\% \text { cash flow }\end{array}$ & $\begin{array}{r}49087 \\
47 \\
\end{array}$ & $\begin{array}{r}116440 \\
94 \\
\end{array}$ & $\begin{array}{r}32565 \\
14 \\
\end{array}$ & $\begin{array}{r}95971 \\
72 \\
\end{array}$ & $\begin{array}{r}76923 \\
54 \\
\end{array}$ \\
\hline $\begin{array}{l}\text { Disponible (I) } \\
\% \text { cash flow }\end{array}$ & $\begin{array}{r}70791 \\
67 \\
\end{array}$ & $\begin{array}{r}17179 \\
14 \\
\end{array}$ & $\begin{array}{r}147533 \\
64 \\
\end{array}$ & $\begin{array}{r}79252 \\
59 \\
\end{array}$ & $\begin{array}{r}70953 \\
50 \\
\end{array}$ \\
\hline $\begin{array}{l}\text { Nouveaux investissements } \\
\text { Nouveaux emprunts } \\
\text { (sauf "reprise") (2) }\end{array}$ & $\begin{array}{r}51876 \\
34620 \\
\end{array}$ & 151161 & 77000 & 68924 & 61529 \\
\hline Disponible (II) & 53535 & 111525 & 125403 & 108499 & 94176 \\
\hline
\end{tabular}

(1) L.M.T. + C.T. + Foncier + Diminution des O.C.C. et dettes (y compris L.M.T. reprise) (voir tableau 6 ).

(2) L.M.T. + C.T. + Augmentation des O.C.C. et dettes (voir tableau 6). Les L.M.T. "reprise" ne sont pas inclus ici puisqu'ils servent à acquérir du capital déjà existant.

de près de $250 \mathrm{~F}$ par ha, en partie du fait de la moindre importance des fermages. Le revenu agricole est, au total, près du double que celui obtenu par ces derniers (1280 F par ha). Si on tient compte de la valeur locative des terres en propriété, le revenu du travail et des capitaux propres ne dépasse pas $52000 \mathrm{~F}$ par travailleur, soit $20000 \mathrm{~F}$ de plus que celui des producteurs T18. L'endettement est aussi élevé ( $43 \%$ sur capital réduit) et les remboursements absorbent $72 \%$ du cashflow. Lavancement de certaines ventes avant la fin de l'année (passage de la production de T2a vendus après le $1^{\text {er }}$ janvier à celle de T20 vendus avant) accroît les ventes dans l'exercice et fait apparaitre une variation d'inventaire pondérale négative (donc une certaine décapitalisation apparente) qui accroît le disponible I. Les investissements en équipement restent inférieurs aux amortissements et aux réalisations d'emprunts nouveaux dans l'exercice. Ceci conduit à des prélèvements familiaux et une variation de l'épargne (ici positive) assez élevés, résultat à la fois d'un bon revenu, d'une anticipation de certaines ventes et d'un léger accroissement de l'endettement.

Les producteurs de T2a combinent la meilleure marge globale et les plus faibles charges de structure par hectare. Les frais de mécanisation sont un peu inférieurs à la moyenne, du fait de la surface plus étendue et de la moindre proportion des cultures. Les charges foncières sont dans la moyenne mais ce sont les frais financiers réduits qui font la plus grande différence.

Le revenu agricole est le plus élevé ( $1645 \mathrm{~F}$ par ha) et même si la forte proportion de faire-valoir direct réduit le revenu du travail et des capitaux propres, celui-ci reste largement le meilleur avec $72900 \mathrm{~F}$ par UTH, plus du double que celui des producteurs de T18 et de broutards.

L'endettement bien maîtrisé ( $26 \%$ ) réduit les remboursements qui n'absorbent que $14 \%$ du cash-flow. Malgré l'augmentation du cheptel et des stocks, le disponible I reste le plus élevé de tous (147000 F). Les investissements en équipement dépassent largement les amortissements, la croissance se poursuit, mais ils sont financés en grande partie par emprunt (ce qui accroît l'endettement là aussi). Et le solde restant pour la famille et l'épargne reste le plus élevé de tous $125400 \mathrm{~F}$, à partager il est vrai entre 2,06 UTH familiales, ce qui ne représente encore que $5070 \mathrm{~F}$ par mois).

\section{Conclusions}

Une grande variabilité existe autour des moyennes qui viennent d'être analysées, comme l'indique la figure 1 . Cette variabilité montre le danger qu'il y a à raisonner à partir de quelques cas seulement même bien choisis et analysés.

On peut néanmoins observer qu'ici, la dispersion confirme très fortement les différences que révèlent les moyennes, ce qui renforce quelque peu les conclusions que l'on peut émettre. De telles différences individuelles, qui rendent très significatifs les écarts entre moyennes, se rencontrent très rarement dans les différents réseaux d'exploitation qui sont habituellement analysés.

Les différences observées en 1986 vont dans le même sens que celles observées depuis 1981. Elles permettent un constat assez clair.

Incontestablement, la production de jeunes taureaux de boucherie de 2 ans conduit à de meilleurs résultats dans les structures où elle est pratiquée, c'est-à-dire des exploitations de bonne dimension, disposant de maind'œuvre et avec une proportion de cultures céréalières très mesurée. Ce système apparaît bien adapté aux conditions et aux systèmes fourragers de la zone charolaise centrale qui, compte tenu des contraintes, restent basés sur l'herbe. Le niveau d'intensification (1,24 UGB par ha SFP) apparaît déjà élevé, ce qui traduit la capacité du système à offrir des possibilités d'intensifications fourragère et zootechnique. Néanmoins, le système exige, pour être réussi, une bonne technicité de la conduite des animaux, au pâturage comme pendant l'hiver.

Les résultats de la production de T20 apparaissent un peu moins bons. Compte tenu de l'échantillon, cela pro- 
vient d'une exploitation nouvelle, qui emploie beaucoup trop de concentré et qui obtient un revenu très légèrement négatif. Les trois autres élevages ont des revenus proches de ceux des T2a. Il faudra poursuivre l'observation de ce système, plus rare, mais qui apparaît aussi très intéressant. Il a notamment l'avantage d'offrir à la commercialisation un étalement de la production en complément de celle des T2a. Mais il est sans doute à réserver aux éleveurs ayant les meilleurs niveaux techniques.

La production de broutards a été particulièrement pénalisée au cours de cette campagne, du fait des sécheresses successives. Les veaux ont été légers malgré des dépenses de concentré importantes. Ceux qui engraissent leurs femelles ont eu aussi des difficultés pour finir leurs génisses de 30 mois à l'herbe, et les prix ont baissé. Néanmoins, ce résultat s'inscrit dans une tendance plus longue observée dans cet échantillon (et en Nièvre) : la production de broutard conduit souvent en Charolais, sauf exception (figure 1) à des revenus inférieurs à ceux procurés par les cycles moyens (Liénard et Lherm 1986). La situation de montagne accentue le phénomène.

L'engraissement des taurillons à l'auge semble mal adapté aux conditions et aux systèmes fourragers possibles dans ces zones. Certes, quelques exploitations arrivent à des résultats moyens (figure 1), mais la plupart ont des revenus faibles. Et sur les 5 exploitations ayant des revenus négatifs, 3 produisent du T18. Cela provient des charges de concentré et de production fourragère trop lourdes, ce que ne compense pas le plus haut produit obtenu, et la marge par UGB n'est finalement pas supérieure à celle des autres systèmes. Mais le phénomène le plus marquant est le plus faible chargement obtenu malgré l'importance donnée au maïs fourrage. L'herbe est sous-exploitée. On accorde trop d'importance au maïs et pas assez à l'herbe. Le cheptel est insuffisant, limité par la trop grande proportion de vaches qu'il faut tenir dans le troupeau et le travail qu'occasionnent les taurillons au printemps et en été. L'importance donnée aux céréales dont les marges sont très moyennes mais qui exigent du matériel contribue aussi à réduire le revenu.

Compte tenu de la nécessité d'avoir des jeunes bovins à commercialiser toute l'année, les trois productions T18, T20, T2a, sont en réalité complémentaires. Le choix doit donc être fait en fonction des éleveurs et des conditions des exploitations ; l'étude suggère en cela un certain nombre d'éléments d'orientation. L'engraissement des taurillons à l'auge doit être réservé aux exploitations de dimensions moyennes, les mieux placées dans la zone pour produire régulièrement du maiis fourrage en bonne condition de rendement et de qualité, ce qui suppose de réduire un peu l'importance donnée aux céréales, sans pour autant négliger l'herbe. Une meilleure connaissance des rendements réellement obtenus dans les exploitations est indispensable. Finalement, une association commerciale de plusieurs zones de production aux potentialités complémentaires ne serait-elle pas, peutêtre, une des solutions à envisager ?

\section{A l'avenir?}

Tous les revenus sont en baisse depuis plusieurs années. En 1986, les revenus par travailleur sont inférieurs de $60 \%$, en francs constants, à ceux de 1981 . Résultat conjugué des deux sécheresses et d'une conjoncture médiocre pour les producteurs de viande bovine. Cela contribue à affaiblir le potentiel de đéveloppement des exploitations. Il ne faut pas s'étonner de la stagnation du cheptel allaitant que l'on constate ici comme ail-

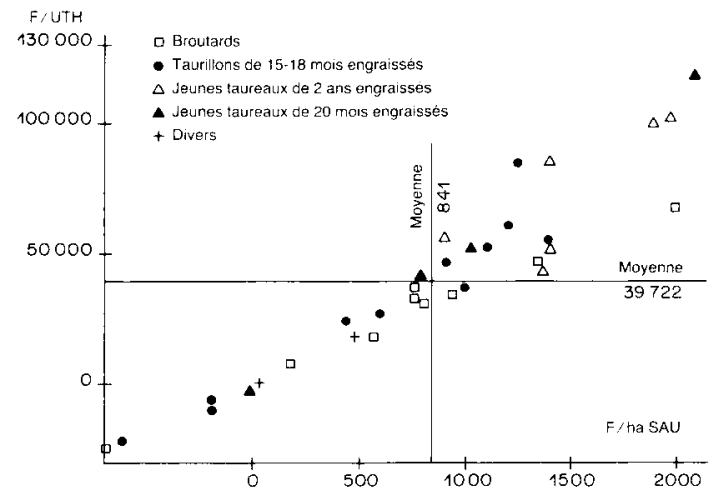

Figure 1. Résultats économiques des 32 élevages allaitants charolais de la Creuse. Revenu du travail et des capitaux propres d'exploitation (Année 1986). leurs. La campagne 1987 climatiquement lavorable aurait pu permettre un début de rétablissement. Mais l'évolution que l'on constate fin 1987-début 1988 ne vas pas le faciliter. En outre, elle peut contribuer à modifier les conclusions qui se dégagent de nos observations sur plusieurs années. En effet, la suppression de l'emploi des anabolisants va rendre beaucoup plus coûteux et plus difficile à réussir l'engraissement des femelles, point fort de ce groupe d'exploitations. Surtout la diminution des cours des jeunes bovins qui survient (notamment en conséquence du changement des règles de l'intervention) va affecter directement les deux systèmes de production T20 et T2a, et sans doute aussi l'engraissement des T18 si cette tendance se maintient. De plus, la production de jeunes bovins laitiers en période hivernale commence à concurrencer celle des zones allaitantes à cette période jusqu'alors favorable. En revanche, la demande en broutard contribue à augmenter leurs cours. Les résultats de 1987 en sont déjà certainement affectés. Sera-ce temporaire ? Toute la question est bien de savoir comment ces évolutions vont se poursuivre dans les années qui viennent. Incontestablement, le niveau des cours des jeunes bovins issus des troupeaux allaitants est déterminant pour l'avenir de cette production dans les zones herbagères défavorisées. On risque fort de voir s'accroître, davantage encore, l'importance de la production d'animaux maigres dans ces régions.

\section{Remerciements}

Nous remercions les éleveurs et les responsables de la Coopérative des Producteurs de Bovins de la Creuse pour leur participation, ainsi que les ingénieurs des Services Techniques de la Chambre d'Agriculture de la Creuse (Gestion et E.D.E.) pour l'aide qu'ils nous apportent.

\section{Références bibliographiques}

GEAY Y., 1986. La production de viande de taurillons. In D. Micol ed. Production de Viande Bovine, INRA Paris, pp. 151-168.

LHERM M., BEBIN D., LIENARD G., 1987. Baisse des revenus et difficultés financières pour les éleveurs allaitants des zones herbagères charolaises. Situation 1985 et évolution récente d'un groupe d'élevages de la Nièvre et de la Creuse. Bull. Techn. CRZV Theix, INRA (67), 59-86.

LIENARD G., LHERM M., 1986. Bases économiques des choix d'un type de production de viande bovine. Cas des troupeaux allaitants. In D. Micol ed. Production de viande bovine, INRA Paris, pp. 273-330.

LIENARD G., LHERM M., BEBIN D., 1987. Incidence économique possible de la suppression de l'emploi des anabolisants pour les éleveurs bovins allaitants des zones herbagères. Bull. Techn. CRZV Theix, INRA (69), 67-75.

MALTERRE C., 1986. Production de viande de génisses. In D. Micol ed. Production de Viande Bovine, INRA Paris, pp. 201-246.

MICOL D., 1986. Production de viande de boufs et jeunes taureaux. In D. Micol ed. Production de Viande Bovine, INRA Paris, pp. 169-200. 
Annexe 1. Les moyens de productions et l'utilisation de la surface fourragère.

\begin{tabular}{|c|c|c|c|c|c|}
\hline (nombre d'exploitations) & $\begin{array}{c}\text { Broutards } \\
\text { (9) }\end{array}$ & $\begin{array}{l}\text { Taurillons } \\
18 \text { mois } \\
\text { Gras } \\
\text { (11) }\end{array}$ & $\begin{array}{l}\text { Taureaux } \\
2 \text { ans } \\
\text { Gras } \\
(6)\end{array}$ & $\begin{array}{l}\text { Taureaux } \\
20 \text { mois } \\
\text { Gras } \\
(4)\end{array}$ & $\begin{array}{l}\text { Moyenne } \\
\text { du groupe } \\
\text { Creuse } \\
\text { (32) }\end{array}$ \\
\hline \begin{tabular}{l|}
$1 /$ La terre \\
S.A.U. \\
Fermage + achat herbe \\
Céréales + A.C.V. (1) \\
S.T.H. \% S.F.P. \\
\end{tabular} & $\begin{array}{l}72 \\
24,4 \\
10,5 \\
60,0\end{array}$ & $\begin{array}{l}94 \\
61,7 \\
21,2 \\
50,2\end{array}$ & $\begin{array}{r}101 \\
36,2 \\
12,0 \\
36,4 \\
\end{array}$ & $\begin{array}{r}80 \\
10,6 \\
9,2 \\
60,9\end{array}$ & $\begin{array}{l}89 \\
41,0 \\
15,2 \\
51,3\end{array}$ \\
\hline $\begin{array}{l}2 / \text { Le travail } \\
\text { U.T.H. } \\
\text { Salariés }\end{array}$ & $\begin{array}{l}1,83 \\
7,6 \\
\end{array}$ & $\begin{array}{l}1,97 \\
9,6\end{array}$ & $\begin{array}{l}2,09 \\
1,4\end{array}$ & $\begin{array}{l}1,46 \\
2,1 \\
\end{array}$ & $\begin{array}{l}1,97 \\
6,6\end{array}$ \\
\hline $\begin{array}{l}3 \text { / Le capital (2) (F/ha S.A.U.) } \\
\text { Animaux } \\
\text { Matériaux } \\
\text { Bâtiments }\end{array}$ & $\begin{array}{ll}8 & 716 \\
2 & 594 \\
2 & 153\end{array}$ & $\begin{array}{ll}7 & 615 \\
2 & 244 \\
2 & 181\end{array}$ & $\begin{array}{l}9419 \\
2742 \\
2298\end{array}$ & $\begin{array}{ll}8 & 802 \\
2 & 722 \\
1 & 214\end{array}$ & $\begin{array}{ll}8 & 433 \\
2 & 531 \\
2 & 091\end{array}$ \\
\hline Total & 13464 & 12041 & 14460 & 12738 & 13056 \\
\hline $\begin{array}{l}4 \text { / Le troupeau } \\
\text { U.G.B. bovins } \\
\text { dont : U.G.B. vaches (3) } \\
\text { U.G.B. élèves mâles } \\
\text { U.G.B. ovins } \\
\end{array}$ & $\begin{array}{r}81,5 \\
46,2 \\
4,5 \\
0,1 \\
81,9\end{array}$ & $\begin{array}{r}82,5 \\
39,9 \\
14,0 \\
1,8 \\
84,4\end{array}$ & $\begin{array}{r}107,1 \\
46,7 \\
24,5 \\
1,3 \\
109,0\end{array}$ & $\begin{array}{r}85,1 \\
38,9 \\
18,6 \\
0,5 \\
85,6 \\
\end{array}$ & $\begin{array}{r}89,1 \\
43,8 \\
14,0 \\
1,7 \\
91,0\end{array}$ \\
\hline $\begin{array}{l}5 \text { / Productivité physique du travail } \\
\text { S.A.U. / U.T.H. (ha) } \\
\text { Nb U.G.B. / U.T.H. } \\
\text { Nb vaches / U.T.H. }\end{array}$ & $\begin{array}{l}39,7 \\
44,3 \\
29,0\end{array}$ & $\begin{array}{l}47,4 \\
43,1 \\
23,4\end{array}$ & $\begin{array}{l}49,0 \\
53,1 \\
26,1\end{array}$ & $\begin{array}{l}54,0 \\
59,3 \\
30,7\end{array}$ & $\begin{array}{l}45,7 \\
47,1 \\
26,2\end{array}$ \\
\hline $\begin{array}{l}\text { / } \text { Maïs ensilage } \\
\text { Nombre d'exploitations en faisant } \\
\text { Surface moyenne (ha) (5) } \\
\text { Surface \% S.F.P. (6) }\end{array}$ & $\begin{array}{l}6 \\
3,3 \\
4,9\end{array}$ & $\begin{array}{c}11 \\
9,6 \\
13,0\end{array}$ & $\begin{array}{l}6 \\
6,3 \\
7,1\end{array}$ & $\begin{array}{c}4 \\
10,6 \\
14,5\end{array}$ & $\begin{array}{r}29 \\
7.8 \\
10,1\end{array}$ \\
\hline $\begin{array}{l}\text { Herbe ensilée } \\
\text { Nombre d'exploitations en faisant } \\
\text { Surface moyenne (ha) (5) } \\
\text { Surface \% S.F.P. (6) }\end{array}$ & $\begin{array}{r}6 \\
6,9 \\
10,1\end{array}$ & $\begin{array}{c}5 \\
12,5 \\
15,3\end{array}$ & $\begin{array}{l}2 \\
4,0 \\
4,0\end{array}$ & $\begin{array}{r}2 \\
8,2 \\
13,9\end{array}$ & $\begin{array}{r}16 \\
8,6 \\
11,0\end{array}$ \\
\hline $\begin{array}{l}\text { Ensemble surface récoltée } \\
\text { Foin \% S.F.P. (5) } \\
\text { Surface récoltée développée } \\
\% \text { S.F.P. (6) (7) }\end{array}$ & $\begin{array}{l}31,6 \\
43,0\end{array}$ & $\begin{array}{l}26,7 \\
47,3\end{array}$ & $\begin{array}{l}38,4 \\
42,7\end{array}$ & $\begin{array}{l}26,4 \\
46,6\end{array}$ & $\begin{array}{l}29,2 \\
44,7\end{array}$ \\
\hline
\end{tabular}

(1) A.C.V. : Autres cultures de vente (maïs grain, colza, etc...)

2) Capital "réduit"'

3) Vaches Charolaises : 0,86 U.G.B. (1 avec le veau jusqu'à 9 mois) ; vaches laitières : 0,90 U.G.B.

(4) Bovins + ovins + chevaux

(5) Par exploitation en ayant.

(6) Moyenne ponderee pour ceux en ayan

(7) Foin + ensilage (herbe + maiis), $1^{1 c}+2^{e}$ coupes

Annexe 2. Résultats techniques du troupeau bovin. Productivité en veau, vêlages et réformes.

\begin{tabular}{|c|c|c|c|c|c|}
\hline (nombre d'exploitations) & $\begin{array}{c}\text { Broutards } \\
\text { (9) }\end{array}$ & $\begin{array}{c}\text { Taurillons } \\
18 \text { mois } \\
\text { Gras } \\
(11)\end{array}$ & $\begin{array}{c}\text { Taureaux } \\
2 \text { ans } \\
\text { Gras } \\
(6)\end{array}$ & $\begin{array}{c}\text { Taureaux } \\
20 \text { mois } \\
\text { Gras } \\
(4)\end{array}$ & $\begin{array}{l}\text { Moyenne } \\
\text { du groupe } \\
\text { Creuse } \\
\text { (32) }\end{array}$ \\
\hline $\begin{array}{l}\text { A / Productivité en veau (1) (\%) } \\
\text { Taux de gestation } \\
\text { Taux de prolificité } \\
\text { Mortalité des veaux } \\
\text { Veaux produits \% vaches vêlées } \\
\text { Productivité numérique } \\
\text { Proportion de vaches } \\
\text { improductives } \\
\text { Productivité globale : nés } \\
\end{array}$ & $\begin{array}{r}96,5 \\
102,6 \\
5,0 \\
97,4 \\
93,1 \\
\\
4,0 \\
89,4 \\
89,4\end{array}$ & $\begin{array}{r}96,7 \\
104,4 \\
8,4 \\
96,4 \\
92,3 \\
\\
3,8 \\
88,7 \\
89,4\end{array}$ & $\begin{array}{r}95,2 \\
104,4 \\
6,0 \\
98,5 \\
92,3 \\
\\
5,4 \\
87,3 \\
87,9\end{array}$ & $\begin{array}{r}95,8 \\
102,7 \\
5,9 \\
98,3 \\
92,6 \\
\\
4,2 \\
88,7 \\
90,2\end{array}$ & $\begin{array}{r}96,3 \\
103,6 \\
6,4 \\
97,5 \\
92,9 \\
\\
4,4 \\
88,8 \\
89,3\end{array}$ \\
\hline 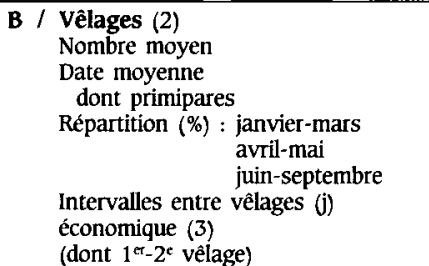 & $\begin{array}{c}55 \\
10 / 03 \\
14 / 02 \\
73 \\
22 \\
2 \\
\\
376 \\
(398)\end{array}$ & $\begin{array}{c}47 \\
28 / 02 \\
11 / 02 \\
79 \\
12 \\
2 \\
\\
373 \\
(396)\end{array}$ & $\begin{array}{c}54 \\
18 / 03 \\
28 / 02 \\
68 \\
26 \\
4 \\
\\
375 \\
(395)\end{array}$ & $\begin{array}{c}45 \\
18 / 02 \\
28 / 01 \\
67 \\
11 \\
3 \\
\\
374 \\
(389)\end{array}$ & $\begin{array}{c}51 \\
06 / 03 \\
14 / 02 \\
73 \\
18 \\
2 \\
\\
374 \\
(394)\end{array}$ \\
\hline $\begin{array}{l}\text { c / Réformes (\%) } \\
\text { Taux de réforme } \\
\text { Taux de renouvellement vrai } \\
\text { Taux d'accroissement du troupeau } \\
\text { de mères }\end{array}$ & $\begin{array}{r}20,8 \\
21,3 \\
0,7\end{array}$ & $\begin{array}{r}18,0 \\
23,4 \\
6,9\end{array}$ & $\begin{array}{r}23,9 \\
25,9 \\
\\
2,0\end{array}$ & $\begin{array}{r}24,7 \\
25,6 \\
1,2\end{array}$ & $\begin{array}{r}20,9 \\
23,6 \\
\\
3,5\end{array}$ \\
\hline
\end{tabular}

(1) Ensemble du troupeau (charolais + croisé + laitier) (le troupeau charolais représente plus de $97 \%$ des vêlages).

(2) Charolais seulement.

(3) Comprennent les multipares ayant "sauté" une campagne de vêlage. 
Annexe 3a. Caractéristiques des bovins vendus (principales catégories).

\begin{tabular}{|c|c|c|c|c|c|}
\hline (nombre d'exploitations) & $\begin{array}{c}\text { Broutards } \\
\text { (9) }\end{array}$ & $\begin{array}{l}\text { Taurillons } \\
18 \text { mois } \\
\text { Gras } \\
\text { (11) }\end{array}$ & $\begin{array}{c}\text { Taureaux } \\
2 \text { ans } \\
\text { Gras } \\
(6)\end{array}$ & $\begin{array}{l}\text { Taureaux } \\
20 \text { mois } \\
\text { Gras } \\
(4)\end{array}$ & $\begin{array}{l}\text { Moyenne } \\
\text { du groupe } \\
\text { Creuse } \\
(32)\end{array}$ \\
\hline $\begin{array}{l}\text { Broutards vente d'automne (3) } \\
\text { Nombre (1) } \\
\text { F/tête } \\
\text { (2) Poids vif } \\
\text { F/kg } \\
\text { Age (mois) } \\
\end{array}$ & $\begin{array}{c}189 \\
4749 \\
280 \\
16,91 \\
7,3\end{array}$ & $\begin{array}{c}21 \\
5073 \\
300 \\
16.90 \\
7.9\end{array}$ & 0 & $\begin{array}{c}1 \\
3150 \\
180 \\
17,50 \\
9,8\end{array}$ & $\begin{array}{c}219 \\
4774 \\
282 \\
16,91 \\
7,4\end{array}$ \\
\hline $\begin{array}{l}\text { Broutards vente d'hiver (4) } \\
\text { Nombre (1) } \\
\text { F/tête } \\
\text { (2) Poids vif } \\
\text { F/kg } \\
\text { Age (mois) }\end{array}$ & $\begin{array}{c}53 \\
5276 \\
323 \\
16,29 \\
10,6\end{array}$ & $\begin{array}{l}16 \\
4757 \\
298 \\
16,37 \\
9,3\end{array}$ & $\begin{array}{c}6 \\
4478 \\
270 \\
16,58 \\
11,0\end{array}$ & 0 & $\begin{array}{l}98 \\
5215 \\
323 \\
16,28 \\
10,5\end{array}$ \\
\hline $\begin{array}{l}\text { Taurillons } 18 \text { mois gras (1) } \\
\text { Nombre (1) } \\
\text { F/tete } \\
\text { (2) Poids net } \\
\text { F/kg } \\
\text { Age (mois) }\end{array}$ & $\begin{array}{c}3 \\
8567 \\
376 \\
22,80 \\
18,0 \\
\end{array}$ & $\begin{array}{c}189 \\
8713 \\
383 \\
22,80 \\
16,5 \\
\end{array}$ & $\begin{array}{c}19 \\
7751 \\
334 \\
23,19 \\
15,1\end{array}$ & $\mathcal{O}$ & $\begin{array}{c}213 \\
8600 \\
379 \\
22,79 \\
16,4 \\
\end{array}$ \\
\hline $\begin{array}{l}\text { Jeunes taureaux de boucherie de } 20 \text { mois (6) } \\
\text { Nombre (1) } \\
\text { F/tete } \\
\text { (2) Poids net } \\
\text { F/kg } \\
\text { Age (mois) }\end{array}$ & o & $\begin{array}{c}1 \\
9108 \\
396 \\
23,00\end{array}$ & 0 & $\begin{array}{c}71 \\
9239 \\
402 \\
23,08 \\
20,6 \\
\end{array}$ & $\begin{array}{l}74 \\
9172 \\
400 \\
23,07 \\
20,6\end{array}$ \\
\hline $\begin{array}{l}\text { Jeunes taureaux de boucherie de } 2 \text { ans } \\
\text { Nombre (1) } \\
\text { F/tête } \\
\text { (2) Poids net } \\
\text { F/kg } \\
\text { Age (mois) } \\
\end{array}$ & $\begin{array}{c}1 \\
8382 \\
381 \\
22,00 \\
20,3\end{array}$ & $\begin{array}{c}19 \\
9024 \\
398 \\
22,64 \\
20,7\end{array}$ & $\begin{array}{c}116 \\
9460 \\
409 \\
23,08 \\
23,6 \\
\end{array}$ & $\begin{array}{c}29 \\
8934 \\
395 \\
22,59 \\
22,7 \\
\end{array}$ & $\begin{array}{c}166 \\
9305 \\
405 \\
22,95 \\
23,3\end{array}$ \\
\hline $\begin{array}{l}\text { Vaches de réforme } \\
\text { Toutes (1) - Nombre } \\
\text { F/tête } \\
\text { dont Grasses - Nombre } \\
\text { Poids net } \\
\text { F/kg } \\
\end{array}$ & $\begin{array}{c}95 \\
6968 \\
53 \\
366 \\
20.91\end{array}$ & $\begin{array}{c}93 \\
8781 \\
88 \\
392 \\
22,65 \\
\end{array}$ & $\begin{array}{c}74 \\
7677 \\
42 \\
393 \\
21,90 \\
\end{array}$ & $\begin{array}{c}51 \\
9385 \\
48 \\
399 \\
23,77 \\
\end{array}$ & $\begin{array}{c}340 \\
8048 \\
256 \\
387 \\
22,41\end{array}$ \\
\hline $\begin{array}{l}\text { Génisses maigres } \\
\text { Broutardes - } \mathrm{Nb}(1) \\
\text { F/tête } \\
\text { (2) Poids vif } \\
\text { F/kg }\end{array}$ & $\begin{array}{c}10 \\
4046 \\
307 \\
13,53\end{array}$ & $\begin{array}{l}10 \\
3493 \\
269 \\
13,70\end{array}$ & 0 & 0 & $\begin{array}{c}20 \\
3770 \\
288 \\
13,61\end{array}$ \\
\hline $\begin{array}{l}\text { Génisses de } 18 \text { mois - Nb (1) } \\
\text { F/tâte }\end{array}$ & $3 \frac{14}{777}$ & 0 & 1500 & 0 & $\begin{array}{c}19 \\
3704\end{array}$ \\
\hline $\begin{array}{l}\text { Génisses de } 24 \text { mois - Nb (1) } \\
\text { F/tete }\end{array}$ & $\begin{array}{c}5 \\
5800 \\
\end{array}$ & 0 & $\begin{array}{c}5 \\
5960\end{array}$ & $\begin{array}{l}4 \\
5 \\
750\end{array}$ & $\begin{array}{l}25 \\
5390\end{array}$ \\
\hline $\begin{array}{l}\text { Génisses engraissées } \\
24 \text { mois - Nb (1) } \\
\text { F/tête } \\
\text { (2) Poids net } \\
\text { F/kg }\end{array}$ & $\begin{array}{c}17 \\
7091 \\
304 \\
23,52\end{array}$ & $\begin{array}{c}45 \\
7665 \\
325 \\
23,77\end{array}$ & $\begin{array}{c}7 \\
7422 \\
311 \\
23,80\end{array}$ & $\begin{array}{c}15 \\
9164 \\
363 \\
25,24\end{array}$ & $\begin{array}{c}86 \\
7741 \\
327 \\
24,04\end{array}$ \\
\hline $\begin{array}{l}25-32 \text { mois }-\mathrm{Nb}(1) \\
\text { F/tète } \\
(2) \text { Poids net } \\
\text { F/kg }\end{array}$ & $\begin{array}{c}19 \\
9083 \\
361 \\
25,19\end{array}$ & $\begin{array}{c}45 \\
8879 \\
357 \\
24,89\end{array}$ & $\begin{array}{c}19 \\
7969 \\
352 \\
22,66\end{array}$ & $\begin{array}{c}8 \\
8836 \\
350 \\
25,26\end{array}$ & $\begin{array}{c}99 \\
8726 \\
355 \\
24,55\end{array}$ \\
\hline $\begin{array}{l}36 \text { mois }-\mathrm{Nb}(1) \\
\text { F/tete } \\
(2) \text { Poids net } \\
\mathrm{F} / \mathrm{kg}\end{array}$ & 0 & $\begin{array}{c}15 \\
9561 \\
389 \\
24,54\end{array}$ & $\begin{array}{c}8 \\
8758 \\
375 \\
23,32\end{array}$ & $\begin{array}{c}5 \\
9801 \\
365 \\
26,85\end{array}$ & $\begin{array}{c}28 \\
9374 \\
381 \\
24,59\end{array}$ \\
\hline
\end{tabular}

Annexe 3b. Importance relative des ventes des différentes catégories de bovins.

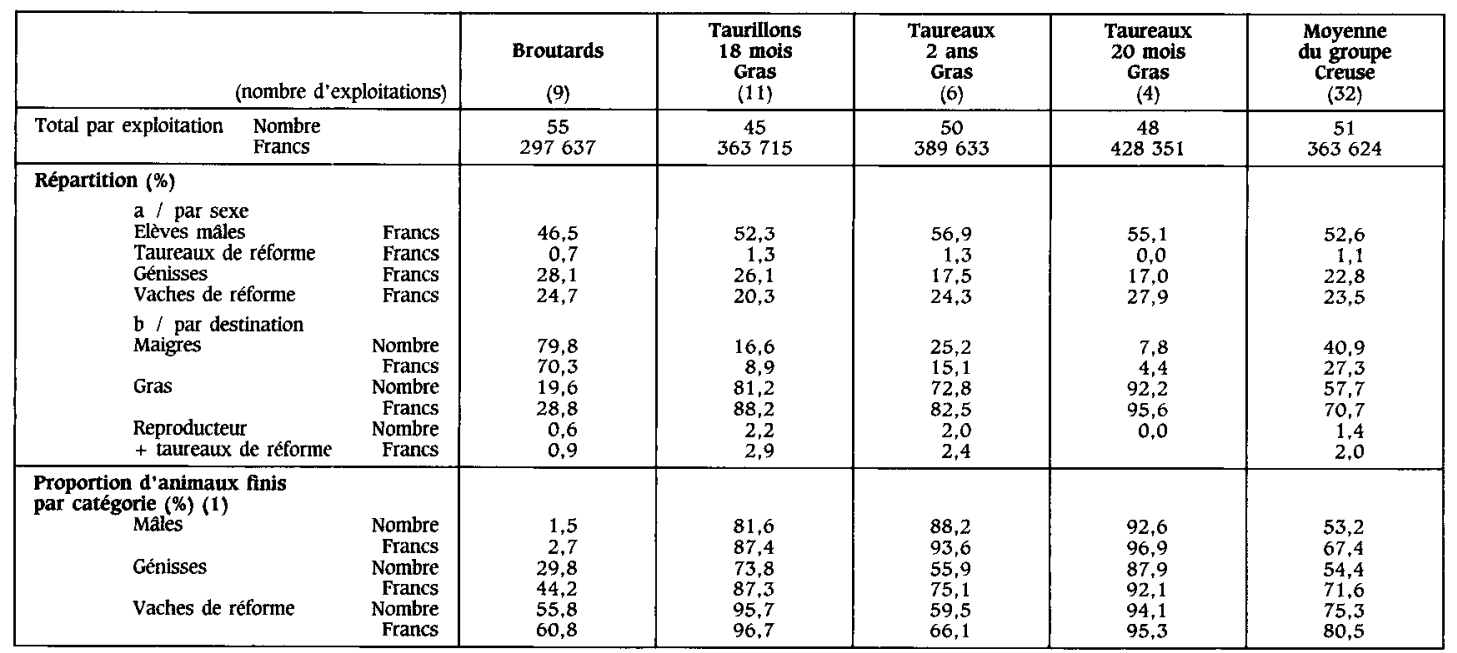

(1) En nombre et en valeur (francs courants). 
Annexe 4. Résultats économiques du troupeau bovin et de la surface fourragère.

\begin{tabular}{|c|c|c|c|c|c|}
\hline (nombre d'exploitations) & $\begin{array}{l}\text { Broutards } \\
\text { (9) }\end{array}$ & $\begin{array}{l}\text { Taurillons } \\
18 \text { mois } \\
\text { Gras } \\
\text { (11) }\end{array}$ & $\begin{array}{c}\text { Taureaux } \\
2 \text { ans } \\
\text { Gras } \\
(6)\end{array}$ & $\begin{array}{l}\text { Taureaux } \\
20 \text { mois } \\
\text { Gras } \\
(4)\end{array}$ & $\begin{array}{l}\text { Moyenne } \\
\text { du groupe } \\
\text { Creuse } \\
(32)\end{array}$ \\
\hline $\begin{array}{l}1 \text { - Résultats du troupeau bovin } \\
\text { En FNGGBB : produit brut }\end{array}$ & 3784 & 4484 & 4069 & 4725 & 4214 \\
\hline $\begin{array}{l}\text { - Variation d'inventaire pondérale } \\
\text { - Ventes nettes } \\
\text { - Primes + aides } \\
\text { - Réévaluation Elèves }\end{array}$ & $\begin{array}{r}-177 \\
3665 \\
294 \\
-17\end{array}$ & $\begin{array}{r}147 \\
4029 \\
267 \\
10\end{array}$ & $\begin{array}{r}420 \\
3362 \\
238 \\
22\end{array}$ & $\begin{array}{r}-431 \\
4812 \\
278 \\
17\end{array}$ & $\begin{array}{r}3 \\
3899 \\
278 \\
6\end{array}$ \\
\hline Marge bovine finale & 2299 & 2642 & 2907 & 2720 & 2591 \\
\hline $\begin{array}{l}\text { Kg viande brute/UGBB } \\
\mathrm{Kg} \text { viande autonome/UGBB } \\
\text { Prix moyen (F/kg vif) (6) }\end{array}$ & $\begin{array}{l}254 \\
201 \\
14,05\end{array}$ & $\begin{array}{l}325 \\
258 \\
13,20\end{array}$ & $\begin{array}{l}293 \\
253 \\
12,99\end{array}$ & $\begin{array}{l}329 \\
246 \\
13,41\end{array}$ & $\begin{array}{l}298 \\
237 \\
13.45\end{array}$ \\
\hline - En F/ha SFPB : marge bovine finale (1) & 2844 & 3075 & 3614 & 3275 & 3136 \\
\hline $\begin{array}{l}\text { - Primes + aides } \\
\text { - Révaluation cheptel élèves }\end{array}$ & $\begin{array}{l}365 \\
-22\end{array}$ & $\begin{array}{r}309 \\
13\end{array}$ & $\begin{array}{r}293 \\
27\end{array}$ & $\begin{array}{r}332 \\
20\end{array}$ & $\begin{array}{r}337 \\
7\end{array}$ \\
\hline $\begin{array}{l}\text { Kg viande brute } / \text { ha SFPB (3) } \\
\text { Kg viande autonome/ha SFPB (4) }\end{array}$ & $\begin{array}{l}317 \\
250 \\
\end{array}$ & $\begin{array}{l}385 \\
301 \\
\end{array}$ & $\begin{array}{l}368 \\
318 \\
\end{array}$ & $\begin{array}{l}398 \\
295 \\
\end{array}$ & $\begin{array}{l}362 \\
287 \\
\end{array}$ \\
\hline $\begin{array}{l}2 \text { - Résultats de la S.F.P. } \\
\text { Par ha SFP Chargement } \\
\mathrm{N}-\mathrm{P}-\mathrm{K} \\
\text { Marge brute SFP (1) (2) } \\
\text { Kg viande autonome }\end{array}$ & $\begin{array}{c}1,24 \\
37-62-34 \\
2845 \\
251 \\
\end{array}$ & $\begin{array}{c}1,16 \\
27-60-39 \\
3128 \\
300 \\
\end{array}$ & $\begin{array}{c}1,24 \\
25-32-27 \\
3611 \\
316 \\
\end{array}$ & $\begin{array}{c}1,20 \\
36-48^{-41} \\
3299 \\
296 \\
\end{array}$ & $\begin{array}{c}1,21 \\
31-53-36 \\
3163 \\
288 \\
\end{array}$ \\
\hline $\begin{array}{l}3 \text { - Charges proportionnelles du troupeau } \\
\text { bovin et de la surface fourragère } \\
\text { Kg d'aliments par UGBB } \\
\text { En } F / U G B B\end{array}$ & 459 & 631 & 344 & 680 & 532 \\
\hline $\begin{array}{l}\text { Aliments concentrés achetés } \\
\text { Aliments concentrés prélevés }\end{array}$ & $\begin{array}{l}385 \\
232\end{array}$ & $\begin{array}{l}454 \\
365\end{array}$ & $\begin{array}{l}231 \\
217\end{array}$ & $\begin{array}{l}721 \\
205\end{array}$ & $\begin{array}{l}281 \\
423\end{array}$ \\
\hline Total aliments concentrés & 617 & 819 & 448 & 926 & 704 \\
\hline Aliments grossiers achetés & 74 & 29 & 46 & 123 & 58 \\
\hline Frais veto & 163 & 203 & 171 & 225 & 185 \\
\hline Autres approvisionnements et frais divers (7) & 140 & 196 & 119 & 184 & 162 \\
\hline Total charges du troupeau & 994 & 1247 & 784 & 1458 & 1109 \\
\hline $\begin{array}{l}\text { - Frais de la } S F P \text { (F/ha SFP) } \\
\text { dont engrais } \\
\text { travail entreprise }\end{array}$ & $\begin{array}{r}618 \\
474 \\
39 \\
\end{array}$ & $\begin{array}{r}700 \\
471 \\
77 \\
\end{array}$ & $\begin{array}{r}478 \\
353 \\
16 \\
\end{array}$ & $\begin{array}{r}645 \\
494 \\
29 \\
\end{array}$ & $\begin{array}{r}626 \\
452 \\
47 \\
\end{array}$ \\
\hline Total charges du troupeau + SFP (F/UGBB) & 1483 & 1842 & 1160 & 2004 & 1622 \\
\hline
\end{tabular}

- Indice francs constants (voir tableau 1)

(1) Frais de culture de la surface fourragère déduits :

2) Ensemble des productions de la S.F.P. (bovins, ovins, chevaux, divers)

(3) Kilo de viande produits (ventes - achats + autoconsommation \pm va
(4) Kilo de viande produits - poids nécessaire pour payer le concentré

(5) Le complément est représenté par les divers (autoconsommation, ventes de lait, récupération sur animaux morts, etc.);

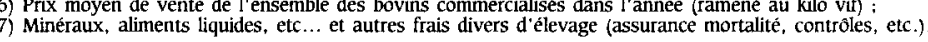

Annexe 5. Résultats économiques globaux.

\begin{tabular}{|c|c|c|c|c|c|}
\hline (nombre d'exploitations) & $\begin{array}{c}\text { Broutards } \\
\text { (9) }\end{array}$ & $\begin{array}{c}\text { Taurillons } \\
18 \text { mois } \\
\text { Gras } \\
(11) \\
\end{array}$ & $\begin{array}{c}\text { Taureaux } \\
2 \text { ans } \\
\text { Gras } \\
(6)\end{array}$ & $\begin{array}{c}\text { Taureaux } \\
20 \text { mois } \\
\text { Gras } \\
(4)\end{array}$ & $\begin{array}{c}\text { Moyenne } \\
\text { du groupe } \\
\text { Creuse } \\
(32)\end{array}$ \\
\hline $\begin{array}{l}\text { 1- Résultats économiques globaux } \\
\text { 1. F/ha SAU } \\
\text { Produit brut d'exploitation } \\
\text { Charges proportionnelles (affectables) } \\
\text { Marge bnute } \\
\text { Charges de structures réelles + (1) } \\
\text { Revenu agricole } \\
\text { Revenu du travail et des capitaux } \\
\text { 2. F/travailleur } \\
\text { Revenu du travail et des capitaux (2) } \\
\text { dont : primes + aides } \\
\text { réévaluation cheptel }\end{array}$ & $\begin{array}{r}4792 \\
1563 \\
3228 \\
2385 \\
844 \\
735 \\
\\
28310 \\
32581 \\
-762\end{array}$ & $\begin{array}{r}5050 \\
1819 \\
3231 \\
2534 \\
698 \\
624 \\
\\
31987 \\
24050 \\
491\end{array}$ & $\begin{array}{rr}5 & 022 \\
1 & 248 \\
3 & 774 \\
2 & 129 \\
1 & 645 \\
1 & 499 \\
& \\
72 & 902 \\
29 & 864 \\
1 & 148\end{array}$ & $\begin{array}{r}5746 \\
2188 \\
3558 \\
2277 \\
1281 \\
987 \\
\\
51 \quad 810 \\
33619 \\
995\end{array}$ & $\begin{array}{r}5035 \\
1672 \\
3363 \\
2399 \\
964 \\
842 \\
\\
39722 \\
28566 \\
315\end{array}$ \\
\hline $\begin{array}{l}\text { 2- Détails de certaines charges réelles } \\
\text { (F/ha SAU) } \\
\text { a) proportionnelles } \\
\text { ' Engrais } \\
\text { - Aliments concentrés achetés } \\
\text { - vétérinaire } \\
\text { b) de structure } \\
\text { - Travail } \\
\text { - Mécanisation } \\
\text { - Batiment } \\
\text { - Foncier } \\
\text { - Divers } \\
\text { - Frais financiers } \\
\text { Total } \\
\text { c) critères spécifiques } \\
\text { - Fermage/ha affermé } \\
\text { - Impôt foncier/ha en propriété } \\
\text { - All. Fam. + Vieillesse/ha SAU (3) }\end{array}$ & $\begin{array}{r}538 \\
599 \\
187 \\
\\
374 \\
793 \\
248 \\
378 \\
358 \\
137 \\
2288 \\
\\
414 \\
167 \\
114\end{array}$ & $\begin{array}{r}644 \\
476 \\
179 \\
\\
364 \\
787 \\
179 \\
539 \\
298 \\
321 \\
2488 \\
\\
531 \\
187 \\
142\end{array}$ & $\begin{array}{r}495 \\
263 \\
181 \\
\\
254 \\
700 \\
230 \\
407 \\
295 \\
149 \\
2035 \\
\\
375 \\
168 \\
124\end{array}$ & $\begin{array}{r}652 \\
843 \\
248 \\
\\
260 \\
772 \\
205 \\
368 \\
266 \\
260 \\
2131 \\
\\
480 \\
194 \\
131\end{array}$ & $\begin{array}{r}582 \\
513 \\
188 \\
\\
342 \\
774 \\
218 \\
449 \\
314 \\
223 \\
2320 \\
\\
487 \\
176 \\
129\end{array}$ \\
\hline
\end{tabular}

(1) Charges de structure réelles + charges proportionnelles non affectables ;
(2) Revenu du travail et capitaux propres d'exploitation = Revenu agricole + salaires et avantages - charge supplétive foncière (valeur locative - impớt foncier) :

Annexe 5 bis. Résultats des cultures de vente.

\begin{tabular}{|c|c|c|c|c|c|}
\hline (nombre d'exploitations) & $\begin{array}{c}\text { Broutards } \\
\text { (9) }\end{array}$ & $\begin{array}{l}\text { Taurillons } \\
18 \text { mois } \\
\text { Gras } \\
\text { (11) }\end{array}$ & $\begin{array}{l}\text { Taureaux } \\
2 \text { ans } \\
\text { Gras } \\
(6)\end{array}$ & $\begin{array}{l}\text { Taureaux } \\
20 \text { mois } \\
\text { Gras } \\
\text { (4) }\end{array}$ & $\begin{array}{l}\text { Moyenne } \\
\text { du groupe } \\
\text { Creuse } \\
\text { (32) }\end{array}$ \\
\hline $\begin{aligned} \text { Céréales : Rendernent (par ha) } \\
\text { Marge brute (par ha) } \\
\text { Céréales + A.C.V. : Marge brute (par ha) } \\
\text { Marge brute (par UTH) }\end{aligned}$ & $\begin{array}{rr} & 30 \\
1 & 806 \\
1 & 806 \\
8 & 200\end{array}$ & $\begin{array}{rr} & 37 \\
2 & 112 \\
2 & 130 \\
22 & 400\end{array}$ & $\begin{array}{rr}32 \\
1744 \\
1785 \\
10000\end{array}$ & $\begin{array}{r}29 \\
1791 \\
1791 \\
10000\end{array}$ & $\begin{array}{r}33 \\
1874 \\
1889\end{array}$ \\
\hline
\end{tabular}




\begin{tabular}{|c|c|c|c|c|c|}
\hline (nombre d'exploitations) & $\begin{array}{c}\text { Broutards } \\
\text { (9) }\end{array}$ & $\begin{array}{c}\text { Taurillons } \\
18 \text { mois } \\
\text { Gras } \\
\text { (11) }\end{array}$ & $\begin{array}{c}\text { Taureaux } \\
2 \text { ans } \\
\text { Gras } \\
\text { (6) }\end{array}$ & $\begin{array}{c}\text { Taureaux } \\
20 \text { mois } \\
\text { Gras } \\
\text { (4) }\end{array}$ & $\begin{array}{c}\text { Moyenne } \\
\text { du groupe } \\
\text { Creuse } \\
(32)\end{array}$ \\
\hline $\begin{array}{l}1 \text { - Capital d'exploitation } \\
\text { (1) (2) En F/exploitation }\end{array}$ & 1007049 & 1051810 & 1462388 & 1028374 & 1146312 \\
\hline $\begin{array}{l}\text { 2- Emprunts (1) } \\
\text { 2.1. Emprunts dexploitation } \\
\text { L.M.T. (F/exploitation) } \\
\text { Reprise } \\
\text { Drainage } \\
\text { C.T. (3) } \\
\text { O.C.C. (Montant 31/12) } \\
\text { Dettes fournisseurs } \\
\text { Total } \\
\text { Taux d'endettement } \\
\text { 2.2. Autres emprunts } \\
\text { Foncier (Flexploitation) } \\
\text { Habitation (F/exploitation) } \\
\text { 2.3. Total endettement }\end{array}$ & $\begin{array}{r}163319 \\
27778 \\
12019 \\
5556 \\
2469 \\
0 \\
211141 \\
21 \\
55503 \\
8790 \\
275434 \\
\end{array}$ & $\begin{array}{r}360105 \\
0 \\
5099 \\
65182 \\
0 \\
6130 \\
436516 \\
41 \\
181890 \\
2874 \\
621280 \\
\end{array}$ & $\begin{array}{r}323191 \\
49722 \\
14466 \\
0 \\
0 \\
0 \\
387379 \\
26 \\
25293 \\
956 \\
413628 \\
\end{array}$ & $\begin{array}{r}428040 \\
0 \\
8979 \\
0 \\
5000 \\
5861 \\
447880 \\
43 \\
252284 \\
46249 \\
746413 \\
\end{array}$ & $\begin{array}{r}314080 \\
17135 \\
8968 \\
23969 \\
1319 \\
2840 \\
368311 \\
32\end{array}$ \\
\hline $\begin{array}{l}\text { 3- Remboursement dans l'exercice } \\
\text { (F/exploitation) } \\
\text { 3.1. L.M.T. (capital) } \\
\text { 3.2. Reprise } \\
\text { 3.3. Drainge } \\
\text { 3.4. C.T. } \\
\text { 3.5. Fonciers (annuités) } \\
\text { Total (3.1. à 3.5.) } \\
\text { (\% du revenu agricole) } \\
\text { 3.6. O.C.C. (variation) (4) } \\
\text { 3.7. Dettes remboursées } \\
\text { Total (3.1. à 3.7.) } \\
\text { (\% du revenu agricole) } \\
\end{array}$ & $\begin{array}{r}22927 \\
0 \\
1121 \\
8333 \\
9618 \\
41999 \\
(74) \\
0 \\
7088 \\
49087 \\
(86) \\
\end{array}$ & $\begin{array}{r}39120 \\
0 \\
254 \\
53727 \\
23339 \\
116440 \\
(160) \\
0 \\
0 \\
116440 \\
(160) \\
\end{array}$ & $\begin{array}{r}27134 \\
824 \\
1208 \\
3399 \\
32565 \\
(20) \\
0 \\
0 \\
32565 \\
(20) \\
\end{array}$ & $\begin{array}{r}29844 \\
0 \\
750 \\
27500 \\
37878 \\
95972 \\
(104) \\
0 \\
0 \\
95972 \\
(104) \\
\end{array}$ & $\begin{array}{r}32042 \\
155 \\
723 \\
24250 \\
17760 \\
74930 \\
(87) \\
0 \\
1993 \\
76923 \\
(90) \\
\end{array}$ \\
\hline $\begin{array}{l}\text { 4- Nouveaux emprunts d'exploitation } \\
\text { contractés dans l'exercice (1) } \\
\text { 4.1. L.M.T. } \\
\text { 4.2. Drainage } \\
\text { 4.3. C.T. } \\
\text { Total } \\
\text { 4.4. O.c.c. (5) } \\
\text { 4.5. Dettes (variations) } \\
\text { Total (4.1. à 4.5.) } \\
\text { 4.6. Reprise } \\
\text { 4.7. Foncier }\end{array}$ & $\begin{array}{r}26777 \\
0 \\
5556 \\
32333 \\
2287 \\
0 \\
34620 \\
27778 \\
0\end{array}$ & $\begin{array}{r}63189 \\
2727 \\
83364 \\
149280 \\
0 \\
1881 \\
151161 \\
0 \\
0\end{array}$ & $\begin{array}{r}77000 \\
0 \\
0 \\
77000 \\
0 \\
0 \\
77000 \\
34667 \\
0\end{array}$ & $\begin{array}{r}63925 \\
0 \\
0 \\
63925 \\
5000 \\
0 \\
68925 \\
0 \\
0\end{array}$ & $\begin{array}{r}52305 \\
937 \\
30219 \\
83461 \\
1268 \\
\quad 23 \\
84752 \\
14312 \\
0\end{array}$ \\
\hline
\end{tabular}

Annexe 6 Endettement.

(1) Situation au 31 décembre de chaque année

(2) Capital réduit = capital cheptel + matériel + bâtiment (non compris les stocks, valeurs en terre et liquidités);

(3) Non compris les dettes fournisseurs et les O.C.C. ;

(5) Augmentation du montant des O.C.C. (sortie - entrée)

\begin{tabular}{|c|c|c|c|c|c|}
\hline (nombre d'exploitations) & $\begin{array}{l}\text { Broutards } \\
\text { (9) }\end{array}$ & $\begin{array}{l}\text { Taurillons } \\
18 \text { mois } \\
\text { Gras } \\
(11)\end{array}$ & $\begin{array}{c}\text { Taureaux } \\
2 \text { ans } \\
\text { Gras } \\
\text { (6) }\end{array}$ & $\begin{array}{c}\text { Taureaux } \\
20 \text { mois } \\
\text { Gras } \\
(4)\end{array}$ & $\begin{array}{c}\text { Moyenne } \\
\text { du groupe } \\
\text { creuse } \\
\text { (32) }\end{array}$ \\
\hline $\begin{array}{l}\text { Revenu agricole } \\
\text { (dont : total aides) } \\
\% \text { cash flow }\end{array}$ & $\begin{array}{r}56869 \\
(59661) \\
54\end{array}$ & $\begin{array}{r}72750 \\
(46647) \\
59\end{array}$ & $\begin{array}{r}164978 \\
\langle 61449\rangle \\
72\end{array}$ & $\begin{array}{r}91176 \\
(48074) \\
68\end{array}$ & $\begin{array}{r}85637 \\
(54533) \\
60\end{array}$ \\
\hline 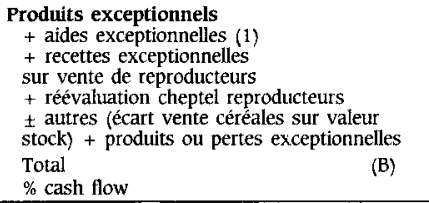 & $\begin{array}{r}6590 \\
5 \\
-6687 \\
662 \\
570 \\
- \\
\end{array}$ & $\begin{array}{r}12710 \\
1627 \\
-5759 \\
1320 \\
9898 \\
8 \\
\end{array}$ & $\begin{array}{r}14504 \\
333 \\
-6660 \\
-131 \\
8046 \\
3 \\
\end{array}$ & $\begin{array}{r}1800 \\
0 \\
-5487 \\
-143 \\
-3830 \\
-3 \\
\end{array}$ & $\begin{array}{r}12014 \\
623 \\
-6286 \\
1042 \\
7393 \\
5\end{array}$ \\
\hline $\begin{array}{l}\text { Ensemble }(A)+(B) \\
\% \text { cash flow }\end{array}$ & $\begin{array}{r}57439 \\
55\end{array}$ & $\begin{array}{r}82648 \\
67\end{array}$ & $\begin{array}{l}173024 \\
75\end{array}$ & $\begin{array}{r}88346 \\
66\end{array}$ & $\begin{array}{r}93030 \\
65 \\
\end{array}$ \\
\hline $\begin{array}{l}\text { Amortissements } \\
\text { \% cash flow } \\
\text { CASH-FLOW }\end{array}$ & $\begin{array}{r}47830 \\
45 \\
105269 \\
\end{array}$ & $\begin{array}{r}41443 \\
33 \\
124091 \\
\end{array}$ & $\begin{array}{r}57530 \\
25 \\
230554 \\
\end{array}$ & $\begin{array}{r}45152 \\
34 \\
133498 \\
\end{array}$ & $\begin{array}{r}49756 \\
35 \\
142786 \\
\end{array}$ \\
\hline $\begin{array}{l}\text { Variations des stocks de production } \\
+ \text { Bovin : - variation pondérale } \\
\text { - réévaluation élèves } \\
\text { - réévaluation reproducteurs } \\
+ \text { Ovin } \\
+ \text { Porc } \\
+ \text { Chevaux } \\
+ \text { Céreales + autres cultures de vente } \\
\text { Total } \\
\% \text { cash flow } \\
\end{array}$ & $\begin{array}{r}-6791 \\
-1364 \\
-6687 \\
-242 \\
-1283 \\
0 \\
-830 \\
-17197 \\
-16 \\
\end{array}$ & $\begin{array}{r}12271 \\
902 \\
-5759 \\
-527 \\
-215 \\
-1182 \\
-5211 \\
279 \\
0 \\
\end{array}$ & $\begin{array}{r}49118 \\
2332 \\
-6660 \\
-427 \\
-69 \\
0 \\
3206 \\
47500 \\
21 \\
\end{array}$ & $\begin{array}{r}-28648 \\
1401 \\
-5487 \\
65 \\
1 \\
0 \\
5168 \\
-27500 \\
-21 \\
\end{array}$ & $\begin{array}{r}5600 \\
604 \\
-6286 \\
22 \\
-448 \\
-406 \\
-925 \\
-1839 \\
-1 \\
\end{array}$ \\
\hline Variation stocks aliments + engrais & 2588 & -9807 & 2956 & -14225 & -3251 \\
\hline Solde & 119878 & 133619 & 180098 & 175223 & 147876 \\
\hline $\begin{array}{l}\text { Remboursements d'emprunts (2) } \\
\% \text { cash flow }\end{array}$ & $\begin{array}{r}49087 \\
47 \\
\end{array}$ & $\begin{array}{r}116440 \\
94 \\
\end{array}$ & $\begin{array}{r}32565 \\
14 \\
\end{array}$ & $\begin{array}{r}95971 \\
72 \\
\end{array}$ & $\begin{array}{r}76923 \\
54 \\
\end{array}$ \\
\hline $\begin{array}{l}\text { DISPONIBLE } \\
\% \text { cash flow }\end{array}$ & $\begin{array}{r}70791 \\
67 \\
\end{array}$ & $\begin{array}{r}17179 \\
14 \\
\end{array}$ & $\begin{array}{r}147533 \\
64 \\
\end{array}$ & $\begin{array}{r}79252 \\
59 \\
\end{array}$ & $\begin{array}{r}70953 \\
50 \\
\end{array}$ \\
\hline $\begin{array}{l}\text { Nouveaux investissements } \\
+ \text { Matériel } \\
\text { + Bâtiments } \\
\text { + Amélioration foncière } \\
\text { - Revente de matériel } \\
\text { Total } \\
\% \text { cash flow }\end{array}$ & $\begin{array}{r}43254 \\
12188 \\
9457 \\
-\quad 13023 \\
51876 \\
62\end{array}$ & $\begin{array}{r}21724 \\
33727 \\
2 \quad 955 \\
-1591 \\
56815 \\
\end{array}$ & $\begin{array}{r}82688 \\
22251 \\
2149 \\
-7958 \\
99130 \\
46\end{array}$ & $\begin{array}{r}40827 \\
0 \\
0 \\
-1150 \\
39677 \\
31\end{array}$ & $\begin{array}{r}43652 \\
19485 \\
4238 \\
-5846 \\
61529 \\
47\end{array}$ \\
\hline Solde après investissement & 18915 & -39636 & 48403 & 39575 & 9424 \\
\hline Nouveaux emprunts (sauf "reprise") (3) & 34620 & 151161 & 77000 & 68924 & 84752 \\
\hline $\begin{array}{l}\text { DISPONIBLE } \\
\% \text { Cash Flow } \\
\% \text { Revenu agricole }\end{array}$ & $\begin{array}{r}53535 \\
51 \\
94\end{array}$ & $\begin{array}{r}111525 \\
90 \\
153\end{array}$ & $\begin{array}{r}125403 \\
54 \\
76\end{array}$ & $\begin{array}{r}108499 \\
81 \\
118\end{array}$ & 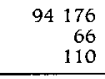 \\
\hline
\end{tabular}

(1) Amortissement des subventions d'équipement + primes orientations viande des Plans de Développement reçues + D.J.A. reçues ;

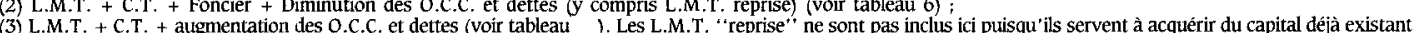
(4) Pour financer l'accroissement des stocks d'approvisionnement (sauf aliments et engrais) + les parts sociales et autres, les variations de liquidite (comptes courants) et les prélèvements familiaux.

Annexe 7. Utilisation du revenu et financement des investissements (en francs par exploitation). 